\title{
Dynamics of action potential firing in electrically connected striatal fast-spiking interneurons
}

\author{
Giovanni Russo, Thierry R. Nieus, Silvia Maggi and Stefano Taverna*
}

Department of Neuroscience and Brain Technologies, Istituto Italiano di Tecnologia, Genoa, Italy

\section{Edited by:}

Enrico Cherubini, International

School for Advanced Studies, Italy

Reviewed by:

Marlene Bartos, University of

Freiburg, Germany

Enrico Bracci, University of

Sheffield, UK

*Correspondence:

Stefano Taverna, Department of

Neuroscience and Brain

Technologies, Istituto Italiano di

Tecnologia, Via Morego 30,

16163 Genoa, Italy

e-mail: stefano.taverna@iit.it
Fast-spiking interneurons (FSIs) play a central role in organizing the output of striatal neural circuits, yet functional interactions between these cells are still largely unknown. Here we investigated the interplay of action potential (AP) firing between electrically connected pairs of identified FSIs in mouse striatal slices. In addition to a loose coordination of firing activity mediated by membrane potential coupling, gap junctions (GJ) induced a frequency-dependent inhibition of spike discharge in coupled cells. At relatively low firing rates $(2-20 \mathrm{~Hz})$, some APs were tightly synchronized whereas others were inhibited. However, burst firing at intermediate frequencies $(25-60 \mathrm{~Hz})$ mostly induced spike inhibition, while at frequencies $>50-60 \mathrm{~Hz}$ FSI pairs tended to synchronize. Spike silencing occurred even in the absence of GABAergic synapses or persisted after a complete block of $\mathrm{GABA}_{A}$ receptors. Pharmacological suppression of presynaptic spike afterhyperpolarization (AHP) caused postsynaptic spikelets to become more prone to trigger spikes at near-threshold potentials, leading to a mostly synchronous firing activity. The complex pattern of functional coordination mediated by GJ endows FSIs with peculiar dynamic properties that may be critical in controlling striatal-dependent behavior.

Keywords: fast-spiking interneurons, striatum, gap junctions, GABA, action potential

\section{INTRODUCTION}

Parvalbumin-expressing, fast-spiking interneurons (FSIs) represent a small fraction of the total cell population in the striatum $(\sim 1 \%$; Luk and Sadikot, 2001), yet they provide a key contribution to sensorimotor integration and functional coordination of striatal network activity (Parthasarathy and Graybiel, 1997; Gage et al., 2010; Tepper et al., 2010; Berke, 2011). FSIs project a feed-forward inhibitory input onto striatal principal cells, the medium spiny neurons (MSNs) (Pennartz and Kitai, 1991; Koos and Tepper, 1999; Gustafson et al., 2006; Mallet et al., 2006; Taverna et al., 2007; Gittis et al., 2010; Planert et al., 2010), and are connected to each other via electrical and GABAergic synapses (Kita et al., 1990; Fukuda, 2009). Several studies demonstrated that gap junctions (GJ), either alone or in coordination with GABAergic synapses, mediate a tight synchronization of FSI spiking and entrainment in gamma-band coherent activity in non-striatal brain areas (Gibson et al., 1999; Tamas et al., 2000; Hormuzdi et al., 2001; Szabadics et al., 2001; Traub et al., 2001; Bartos et al., 2002). In the striatum, FSIs are known to (i) fire repetitive bursts of action potentials (APs) at an intra-burst frequency of $\sim 40-50 \mathrm{~Hz}$ (Bracci et al., 2003; Plotkin et al., 2005; Taverna et al., 2007) and (ii) express functional GJ (Koos and Tepper, 1999). Recent in vivo studies, however, showed that FSIs fire mostly asynchronously during striatal-dependent behavioral tasks (Berke, 2008; Gage et al., 2010) and in certain conditions may exert a direct inhibitory effect onto each other (Lansink et al., 2010). A recent modeling paper suggested that striatal GJ mediate a shunting effect which reduces spike firing in FSI pairs receiving uncorrelated excitatory inputs (Hjorth et al., 2009). Moreover, no significant synchronization was detected in simulated FSI striatal networks connected by both GABAergic synapses and GJ (Humphries et al., 2009). These findings suggest that synaptic connectivity between striatal FSI may be characterized by distinctive properties which promote fast inhibition rather than synchronous firing. Yet, it is unknown how GJ and GABAergic synapses shape the firing activity in live striatal FSIs, therefore a direct assessment of functional communication between these cells is needed. Here, we sought to identify the role played by electrical and GABAergic synapses in coordinating the firing activity of FSI pairs using dual patch-clamp recordings in mouse neostriatal slices. We found that GJ promoted fast, frequency-dependent inhibition of postsynaptic firing along with a slower membrane potential coupling effect which induced a loose firing coherence between simultaneously recorded cells. AP inhibition was induced by the action of the slow hyperpolarizing component of postsynaptic spikelets. Such effects were correlated with the strength of GJ conductance $(\mathrm{Gc})$ and were independent of the presence of GABAergic synapses.

\section{MATERIALS AND METHODS ELECTROPHYSIOLOGY}

Patch-clamp recordings were performed on pairs of genetically identified FSIs in striatal slices prepared from a recombinant Cre-lox mouse line obtained by crossing 129P2-Pvalbtm1(cre)Arbr (JAX stock number: 008069) and 129S6-Gt(ROSA)26Sortm14(CAG-tdTomato)Hze mice (JAX stock number: 007914; Jackson Laboratory, Bar Harbor, ME, USA; Madisen et al., 2010). Offspring mice, which appeared 
viable and healthy, expressed the td-Tomato red fluorescent protein in FSIs throughout the brain (Figure 1). All procedures were approved by the Italian Department of Health and were conducted in accordance to FELASA guidelines as well as Italian and European directives (DL 116/92 and 2010/63/EU). Mice of both sexes (p18-p38, average p25) were anesthetized with an intraperitoneal injection of a mixture of ketamine/xylazine $(100 \mathrm{mg} / \mathrm{kg}$ and $10 \mathrm{mg} / \mathrm{kg}$, respectively) and perfused transcardially with ice-cold artificial cerebrospinal fluid (ACSF) consisting of (in $\mathrm{mM}): 125 \mathrm{NaCl}, 2.5 \mathrm{KCl}, 1.25 \mathrm{NaH}_{2} \mathrm{PO}_{4}, 2 \mathrm{CaCl}_{2}, 25 \mathrm{NaHCO}_{3}$, $1 \mathrm{MgCl}_{2}$, and $11 \mathrm{D}$-glucose, saturated with $95 \% \mathrm{O}_{2}$ and $5 \%$ $\mathrm{CO}_{2}$ ( $\mathrm{pH}$ 7.3). After decapitation, brains were removed from the skull and $300 \mu \mathrm{m}$-thick parasagittal slices were cut in ACSF at $4^{\circ} \mathrm{C}$ using a VT1000S vibratome (Leica Microsystems, Wetzlar,
Germany). Individual slices were submerged in a recording chamber in which ACSF was continuously flowing (1-2 $\mathrm{ml} / \mathrm{min}$ ) at $32^{\circ} \mathrm{C}$. NBQX $(5 \mu \mathrm{M})$ was added to ACSF at the beginning of experiments to block AMPA receptors. Patch clamp glass pipettes (2-4 M $\Omega$ ) contained the following (in $\mathrm{mM}$ ): $10 \mathrm{NaCl}$, $124 \mathrm{KH}_{2} \mathrm{PO}_{4}, 10$ HEPES, 0.5 EGTA, $2 \mathrm{MgCl}_{2}, 2 \mathrm{Na}_{2}$-ATP, 0.02 $\mathrm{Na}-\mathrm{GTP}$, ( $\mathrm{pH} 7.2$, adjusted with $\mathrm{KOH}$ ). For whole-cell recordings in high intracellular chloride conditions and for perforated-patch experiments the following solution was used (in $\mathrm{mM}$ ): $10 \mathrm{NaCl}$, $24 \mathrm{KH}_{2} \mathrm{PO}_{4}, 100 \mathrm{KCl}, 10 \mathrm{HEPES}, 0.5$ EGTA, $2 \mathrm{MgCl}_{2}$, $2 \mathrm{Na}_{2}$-ATP, $0.02 \mathrm{Na}-\mathrm{GTP}$ ( $\mathrm{pH} 7.2$, adjusted with $\mathrm{KOH}$ ). For perforated-patch recordings the intracellular solution was added with gramicidin D (final concentration $5-10 \mu \mathrm{g} / \mathrm{ml}$, from a stock solution of $5 \mathrm{mg} / \mathrm{ml}$ dissolved in DMSO), a ionophore compound which
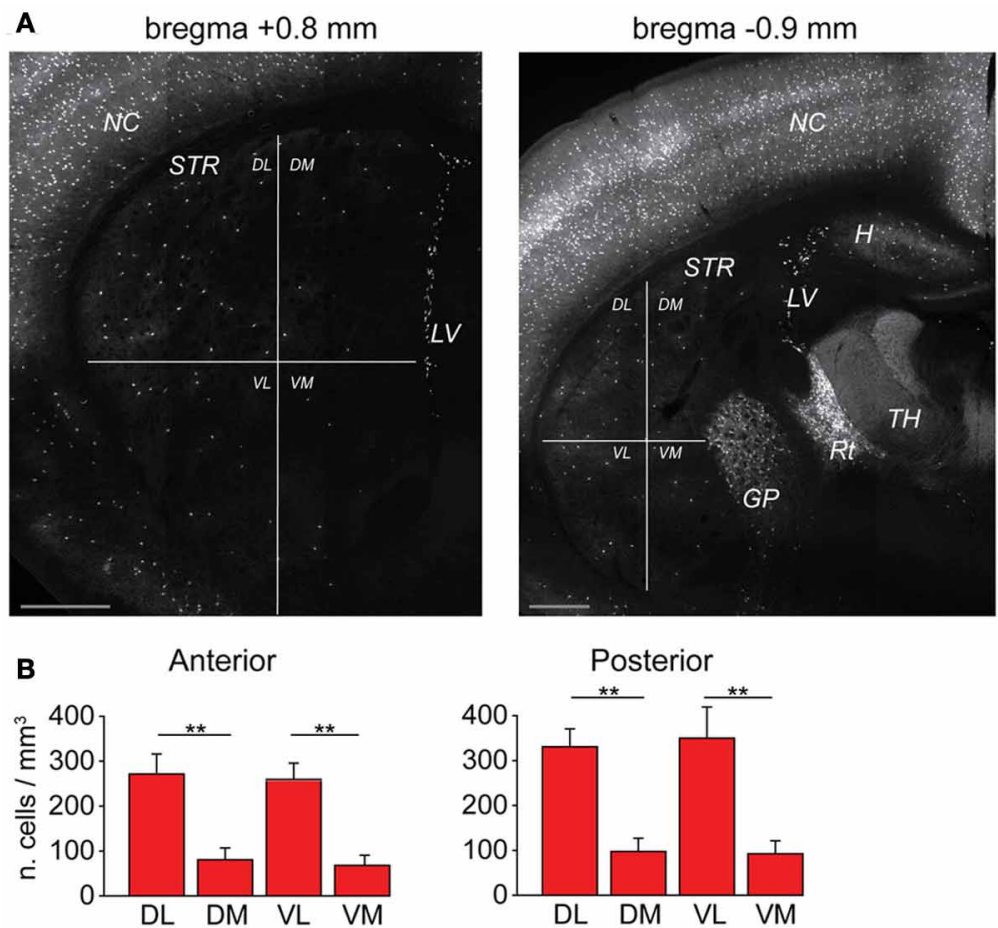

C
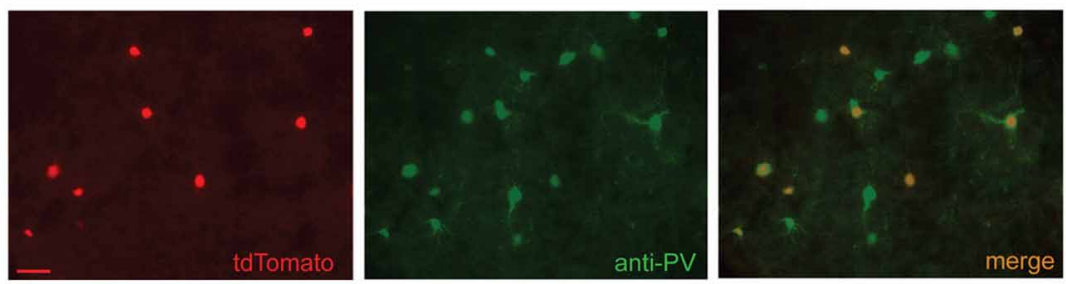

FIGURE 1 | Anatomical distribution of striatal FSIs and specificity of parvalbumin expression. (A) $\mathrm{PV}^{+}$-tdTomato-labeled FSIs in mouse acute slices. FSIs were prevalently distributed in the dorsolateral (DL) and ventrolateral (VL) sectors of the striatum (STR) as compared to dorsomedial (DM) and ventromedial (VM) quadrants. Examples of anterior (left) and posterior (right) $50 \mu \mathrm{m}$-thick striatal sections are shown $(+0.8 \mathrm{~mm}$ and $-0.9 \mathrm{~mm}$ from bregma, respectively). Note the greater density of tdTomato labeled cells in other areas such as neocortex (NC), globus pallidus (GP), reticular thalamic nucleus (Rt) and lateral ventricles (LV), and hippocampus $(\mathrm{H})$, while non-reticular thalamic nuclei $(\mathrm{TH})$ contain densely stained fibers but are almost entirely devoid of tdTomato-labeled cell bodies. Scale bars: $500 \mu \mathrm{m}$. (B) Quantification of FSI distribution in the striatum. Data refer to average cell densities per slice \pm s.e.m. Counts were made in $50 \mu \mathrm{m}$ thick slices ( $n=19$ ) and grouped according to anterior vs. posterior localization of the relative slice with respect to bregma (see Results). Anterior: $n=8$ slices, ${ }^{* *} p<0.01$ paired $t$-test; posterior: $n=11$ slices, ${ }^{* *} p<0.01$. (C) Comparison between expression of td-Tomato (left) and anti-PV immunostaining (middle) in a sample area of DL striatum. An overlap of the two images (right) shows that all td-Tomato cells were also PV-immunopositive, while roughly $50 \%$ of the PV-immunopositive cells did not express tdTomato. Scale bar: $50 \mu \mathrm{m}$ 
creates $\mathrm{Cl}^{-}$-impermeable membrane pores (Ebihara et al., 1995). Recording pipettes were tip-filled with a gramicidin-free solution and back-filled with a gramicidin-containing solution. A stable perforated-patch was achieved after obtaining a gigaohm seal and waiting 30-40 min until the access resistance had gradually reached a stable value $(<30 \mathrm{M} \Omega)$. Capacitive currents were continuously monitored by applying voltage pulses $(-10 \mathrm{mV}$, $500 \mathrm{~ms}$ ) from a holding potential of $-70 \mathrm{mV}$. Recordings were discarded when a sudden membrane rupture occurred as indicated by a quick increase in the amplitude of capacitive transients and a rightward shift in inhibitory postsynaptic current (IPSC) reversal potential. Voltage- and current clamp recordings were performed using a MultiClamp 700B amplifier interfaced to a PC through a Digidata 1440A (Molecular Devices, Sunnyvale, CA, USA). Series resistance was compensated in current clamp recordings using the bridge-balance control of the amplifier software panel.

\section{DATA ACQUISITION AND ANALYSIS}

Data were acquired at a sampling frequency of $10 \mathrm{KHz}$ and filtered at $2 \mathrm{KHz}$ using pClamp10 software (Molecular Devices) and analyzed with Origin 8.5 (Origin Lab, Northampton, MA, USA). Immediately after obtaining a whole-cell configuration in voltage-clamp mode in two cells, recordings were switched to current-clamp and the resting membrane potential was measured. The membrane time constant was calculated by fitting with a single exponential equation the rising trajectory of the membrane voltage response to an injected rectangular current pulse $(-100 \mathrm{pA}, 500 \mathrm{~ms})$. The spike threshold was measured at the time point at which the first derivative of the AP waveform rapidly deflected upwards. The spike width was defined as the time window at half-maximal spike amplitude.

The coupling coefficient (CC) between FSI pairs connected through GJ was calculated after injecting a hyperpolarizing current step $(-300 \mathrm{pA}, 500 \mathrm{~ms})$ in one cell and dividing the steady-state value of the voltage deflection observed in the non-injected cell by that measured in the injected one. Gc was calculated according to the following equation (Galarreta and Hestrin, 2002):

$$
\mathrm{Gc}=1 /\left[\left(R_{\mathrm{in}} / \mathrm{CC}\right)-R_{\mathrm{in}}\right],
$$

where $R_{\text {in }}$ is the input resistance of the non-stimulated cell and $\mathrm{CC}$ is the coupling coefficient. The input resistance was measured in each cell by dividing the steady-state value of the voltage deflection evoked in response to a hyperpolarizing current step $(-100 \mathrm{pA}, 500 \mathrm{~ms})$ by the amplitude of the injected current. The amplitudes of APs and afterhyperpolarizations (AHPs) were measured as the voltage difference between spike positive and negative peak vs. spike threshold, respectively.

Unitary spikelet peak and trough amplitudes were measured in current clamp-mode during firing trains at $30-40 \mathrm{~Hz}$, using the point of slope change in uprise deflection as a baseline reference (Figure 3A, inset). The temporal summation of spikelet hyperpolarizing phases (SHPs) was calculated as the ratio between any largest SHP peak and the first SHP peak during a spikelet train at $10-120 \mathrm{~Hz}$. The reference baseline was the average membrane potential value $\left(\mathrm{V}_{\mathrm{m}}\right)$ across a $5 \mathrm{~ms}$ window preceding the first spikelet. Paired-pulse ratios of unitary GABAergic currents were measured by eliciting two IPSCs at a time interval of $50 \mathrm{~ms}$ and calculating the ratio between the second IPSC peak amplitude and the first one. Since fast GJ currents $\left(\mathrm{I}_{\mathrm{GJ}}\right)$ were partially overlapped to the rising phase of IPSCs in pairs connected through both electrical and GABAergic synapses (Figure 5A, inset), IPSC $10-90 \%$ rise time values were measured only in a subset of pairs which were connected exclusively through GABAergic synapses. Conversely, IPSC peak amplitude and decay time constant values were well separated from $I_{G J}$, therefore these values were collected from both GJ + GABA and GABA-only pairs. The reversal potential for $\mathrm{Cl}^{-}$ions was calculated using the Nernst equation, $E_{\mathrm{Cl}}=-60 \log [\mathrm{Cl}]_{\mathrm{o}} /[\mathrm{Cl}]_{\mathrm{i}}$ at $T=32^{\circ} \mathrm{C}$.

The frequency-dependent temporal summation of SHPs (Figure 3A) was fit using the Boltzmann equation:

$$
y=A 2+\frac{A 1-A 2}{1+e^{\frac{x-x_{0}}{d x}}}
$$

where $A 1$ and $A 2$ represent the left- and rightmost asymptotic values of $y$, respectively, $x_{0}$ is the frequency value corresponding to half-maximal summation, and $d x$ is the rising phase slope.

The degree of covariation in firing activities of connected cells was quantified by means of the Pearson's correlation coefficient (PMFR), which was computed according to equation 3:

$$
P M F R=\frac{\sum_{i}\left(M F R_{1}\left(t_{i}\right)-\overline{M F R_{1}}\right)\left(M F R_{2}\left(t_{i}\right)-\overline{M F R_{2}}\right)}{\sqrt{\sum_{i}\left(M F R_{1}\left(t_{i}\right)-\overline{M F R_{1}}\right)^{2} \sum_{i}\left(M F R_{2}\left(t_{i}\right)-\overline{M F R_{2}}\right)^{2}}}
$$

where $M F R_{1}\left(t_{i}\right)$ and $M F R_{2}\left(t_{i}\right)$ correspond to the time-varying mean firing rate of the two cells measured at the instants $t_{i}$ and $\overline{M F R_{1}}, \overline{M F R_{2}}$ represent the average firing rates. Time-dependent MFRs were computed on sliding time windows of fixed size ( $150 \mathrm{~ms}$ ) stepped by $t_{i}$ intervals of $10 \mathrm{~ms}$. The size of the sliding window was large enough to ensure a reliable estimation of the firing rates and the PMFR. The co-silent instants (i.e., $M F R_{1}\left(t_{i}\right)=$ $\operatorname{MFR}_{2}\left(t_{i}\right)=0$ ) were discarded from the analysis to prevent positive biases of the PMFR when both cells were not firing. PMFR values varied in the interval $[-1,1]$, where values $>0$ indicate positive covariation while values $<0$ indicate negative covariation of the firing activities.

Cross-correlations were computed according to equation 4:

$$
C_{12}(\tau)=\frac{\sum_{t_{i}} X_{1}\left(t_{i}\right) X_{2}\left(t_{i}+\tau\right)-<X_{1} X_{2}>}{\sqrt{N_{1} N_{2}}}
$$

where $t_{i}$ is the spike time of the reference spike train $X_{1}(i=$ $\left.1 . . N_{1}\right)$ with time resolution $1 \mathrm{~ms}$ and the convention $X_{1}\left(t_{i}\right)=1$ or 0 , depending on whether a spike did or did not occur in the time bin $t_{i}$, respectively. $\left\langle X_{1} X_{2}\right\rangle$ is the joint average number of spikes. The normalization factor is the geometric mean of the number of spikes in the two trains $\left(N_{1}\right.$ and $\left.N_{2}\right)$. A threshold for significant cross-correlation was determined as the mean plus two standard deviations of cross-correlation peaks computed by jittering the original spike train (maximal shift equal to the mean inter-spike-interval). PMFR and cross-correlation analyses were performed by means of a custom-made code developed in Python (www.python.org).

Statistical analyses were performed using paired or unpaired Student's $t$-tests for normally distributed data sets, and Wilcoxon 
signed rank test otherwise (SigmaStat; Systat Software, Chicago, IL, USA). Results are given as means \pm s.e.m. in text and represented by box plots in figures. Boxes include 25th and 75th percentiles (horizontal edges), median value (inner line), and min-max values (whiskers). Differences were considered significant at $p<0.05$.

\section{DYNAMIC-CLAMP}

Simulated EPSC conductances (sEPSCs) were injected through the patch pipette using a SM-2 Digital Conductance Injection System (Cambridge Conductances, Cambridge, UK) interfaced through a P25M DSP board (Innovative Integration, Simi Valley, CA, USA). sEPSCs were modeled in Python based on the waveform of real spontaneous EPSCs recorded in FSIs in voltageclamp mode at a $V_{\text {clamp }}$ of $-70 \mathrm{mV}$. The $10-90 \%$ rise time and decay time constant $\left(\tau_{\mathrm{dec}}\right)$ were $0.3 \mathrm{~ms}$ and $0.85 \mathrm{~ms}$, respectively. Peak conductance amplitudes $(6-8 \mathrm{nS})$ were set to values which were suitable to bring $\mathrm{V}_{\mathrm{m}}$ to firing threshold during high-frequency stimulation in current-clamp mode. The reversal potential was set at $0 \mathrm{mV}$.

\section{IMMUNOHISTOCHEMISTRY}

Mice were anaesthetized with ketamine/xylazine $(100 \mathrm{mg} / \mathrm{kg}$ and $10 \mathrm{mg} / \mathrm{kg}$, respectively) and perfused transcardially with $4 \%$ paraformaldehyde in $0.1 \mathrm{M}$ phosphate buffer at $\mathrm{pH}$ 7.4. Brains were quickly removed and placed in fixative overnight at $4^{\circ} \mathrm{C}$. Subsequently they were cryo-protected in $30 \%$ sucrose in $0.1 \mathrm{M}$ phosphate buffer overnight at $4^{\circ} \mathrm{C}$. Brains were rapidly frozen and $50 \mu \mathrm{m}$ thick coronal sections were cut using a Microm HM450 sliding microtome (Thermo Fisher Scientific, Waltham, MA, USA). Sections were rinsed three times with $1 \%$ TBS and stored for $2 \mathrm{~h}$ in a blocking solution $(1 \%$ TBS $+0.5 \%$ Triton $+1 \% \mathrm{BSA}+5 \% \mathrm{NGS})$ at $4^{\circ} \mathrm{C}$. Eventually, sections were incubated with a rabbit polyclonal anti-parvalbumin (PV) antibody (1:600) overnight at $4^{\circ} \mathrm{C}$, rinsed three times in $1 \%$ TBS $+0.1 \%$ Triton, and subsequently incubated with a goat anti-rabbit Alexa 488-conjugated secondary antibody (1:200; Life Technologies, Monza, Italy) for $3 \mathrm{~h}$ in a dark room at $4^{\circ} \mathrm{C}$. Sections were then mounted in Vectashield (Vector Laboratories, Peterborough, UK) and examined with a fluorescence BX51 microscope (Olympus, Japan) equipped with a reconstruction software (Neurolucida, MBF Bioscience, Magdeburg, Germany). For each slice the striatum was subdivided in four sectors (dorso- and ventrolateral, dorso- and ventromedial) and the volume of each sector was measured using the Neurolucida image reconstruction system. Fluorescent FSIs were counted in every sector across three sequential focal planes (each of $15 \mu \mathrm{m}$ along the $\mathrm{Z}$ axis) acquired using confocal laser scanning. Cell densities were obtained by dividing the number of FSIs by the volume of the relative sector.

For cell reconstruction the intracellular solution was added with Neurobiotin 488 tracer $(1.5 \mathrm{mg} / \mathrm{ml}$; Vector Laboratories, Peterborough, UK). Individual FSIs were patch-clamped in whole-cell configuration and their firing pattern was recorded in current-clamp mode. After recording, slices $(300 \mu \mathrm{m}$ of thickness) were fixed overnight at $4^{\circ} \mathrm{C}$ in $4 \%$ paraformaldehyde in $0.1 \%$ phosphate buffer ( $\mathrm{pH} 7.4$ ). Eventually, sections were rinsed three times with $4 \%$ PBS, mounted in Vectashield, and examined with a confocal SP5 upright microscope (Leica, Germany).

\section{DRUGS}

All drugs were obtained from Sigma except NBQX, SR95531, and CGP52432 (Abcam Biochemicals, Bristol, UK).

\section{RESULTS}

In order to visualize PV-expressing interneurons we used striatal slices obtained from transgenic mice in which the fluorescent protein tdTomato was expressed under the promoter for PV (see Methods). FSIs were visible in the striatum as a relatively sparse population of tdTomato-labeled cells (Figure 1A) significantly more abundant in lateral than medial striatal areas, both anteriorly and posteriorly (up to $+1.2 \mathrm{~mm}$ and $-1.2 \mathrm{~mm}$ from bregma, respectively; Figure 1B) consistently with previous reports (Gerfen et al., 1985; Kita et al., 1990; Mura et al., 2000; Luk and Sadikot, 2001). After immunohistochemical staining with an anti-PV antibody, the fraction of labeled cells which also expressed tdTomato was $40 \pm 3 \%$ of the total anti-PV labeled cells $\left(238\right.$ anti-PV $^{+} /$tdTomato $^{+}$vs. 602 anti-PV $^{+} /$tdTomato $^{-}, n=19$ slices from 4 mice), while $100 \%$ of tdTomato-expressing cells were labeled by anti-PV antibodies (Figure 1C). The high specificity of the reporter gene expression was confirmed by the electrophysiological properties of recorded cells, which invariably displayed typical patterns of FSIs (see below). For recordings, we used cell pairs located mainly in the anterior or posterior dorsolateral portion of the striatum. No significant differences were found between electrical properties or connectivity patterns of FSIs located in different striatal territories, therefore data were pooled together. Simultaneous whole-cell patch clamp recordings were performed in pairs of tdTomato-labeled FSIs. Intrinsic membrane and spike properties of these cells are summarized in Table 1. In current-clamp experiments most FSIs responded to injection of relatively brief, strong current pulses $(700 \mathrm{pA}, 500 \mathrm{~ms})$ with a regular train of AP at a mean frequency of $132 \pm 2 \mathrm{~Hz}$ (Figure 2A, inset). To detect steady-state firing patterns in a subset of FSIs we injected prolonged suprathreshold DC $\left(\mathrm{I}_{\mathrm{inj}}: 400-800 \mathrm{pA}\right.$, 20-150 s; Figure 2A). Most cells (140 out of 263, 53\%) responded with repetitive bursts of AP with a mean intra-burst frequency

Table 1 | Electrophysiological properties of striatal FSIs.

\begin{tabular}{lccc}
\hline & $\begin{array}{c}\text { Bursting } \\
(\boldsymbol{n}=\mathbf{1 4 0})\end{array}$ & $\begin{array}{c}\text { Irregular } \\
(\boldsymbol{n}=\mathbf{7 7})\end{array}$ & $\begin{array}{c}\text { Regular } \\
(\boldsymbol{n}=\mathbf{4 6})\end{array}$ \\
\hline Resting membrane potential $(\mathrm{mV})$ & $-74 \pm 0.5$ & $-74 \pm 0.6$ & $-76 \pm 1$ \\
Input resistance $(\mathrm{M} \Omega)$ & $60 \pm 2$ & $63 \pm 3$ & $59 \pm 5$ \\
Membrane time constant (ms) & $9.8 \pm 0.2$ & $9.5 \pm 0.4$ & $9.3 \pm 0.4$ \\
AP peak amplitude (mV) & $60 \pm 1$ & $55 \pm 2$ & $58 \pm 2$ \\
AP threshold (mV) & $-45 \pm 0.6$ & $-43 \pm 0.5$ & $-46 \pm 1$ \\
AP width (ms) & $0.54 \pm 0.01$ & $0.56 \pm 0.01$ & $0.55 \pm 0.02$ \\
AP time to peak (ms) & $0.44 \pm 0.01$ & $0.46 \pm 0.01$ & $0.46 \pm 0.02$ \\
AHP amplitude (mV) & $15 \pm 0.4$ & $14 \pm 0.5$ & $13 \pm 0.7$ \\
\hline
\end{tabular}

All values are means \pm s.e.m. Input resistance and membrane time constant were measured on passive voltage responses to injection of negative current pulses $(-100 \mathrm{pA}, 500 \mathrm{~ms})$. Action potentials (APS) were elicited by injection of suprathreshold current pulses $(400 \mathrm{pA}, 500 \mathrm{~ms})$. AHP: afterhyperpolarization. AP time to peak and AHP were calculated using the spike threshold as a reference point. 


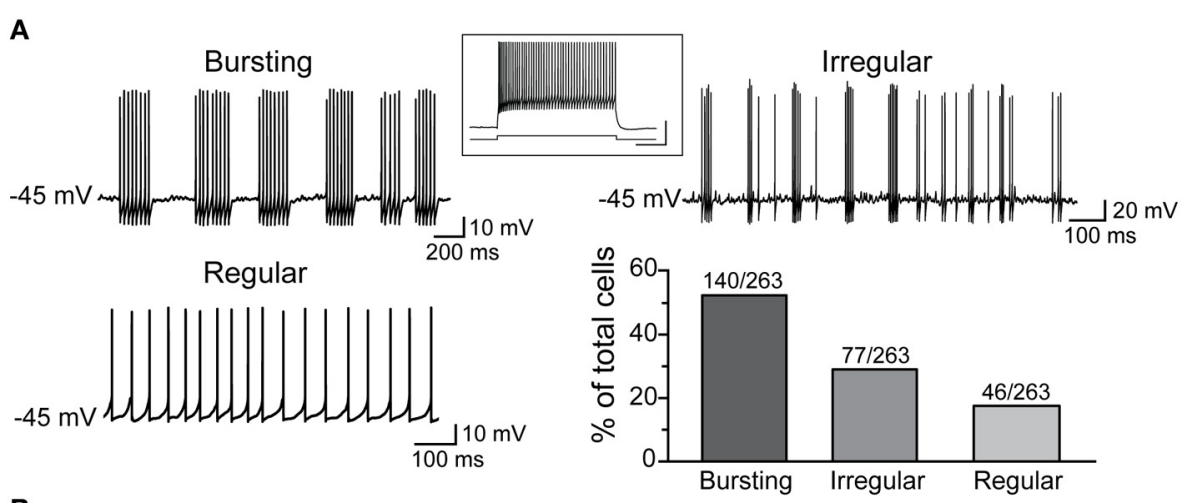

B
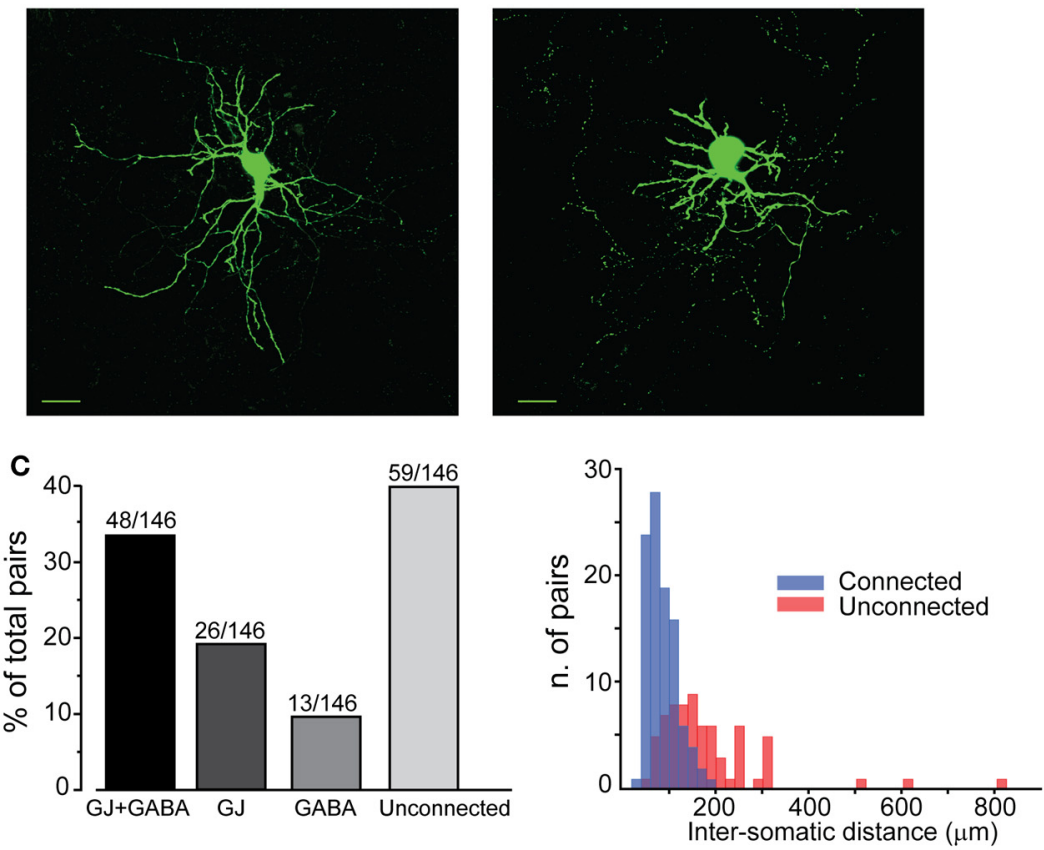

FIGURE 2 | Electrophysiological properties and connectivity patterns of striatal FSIs. (A) Examples of different firing patterns and relative distributions. Traces are excerpts from 20 to $60 \mathrm{~s}$ lasting recordings in which cells were injected with suprathreshold DC ( inj $_{\text {bursting: }}$ b00 pA; regular: $400 \mathrm{pA}$; irregular: $500 \mathrm{pA}$ ). The histogram shows relative distributions of different firing patterns. Inset: high-frequency firing activity in response to supra-threshold current pulse (700 pA, bottom trace). Calibration: $30 \mathrm{mV}, 250 \mathrm{~ms}$. (B) Confocal microphotographs showing two different FSIs filled with neurobiotin-488 (scale bars: $20 \mu \mathrm{m}$ ). (C) Left, rates of different connectivity patterns between pairs of striatal FSIs. Right, spatial distribution of connected and unconnected FSI pairs relative to the distance between cell bodies. of $39 \pm 1 \mathrm{~Hz}$. A minor subset of FSIs (46 out of 263, 18\%) fired in single-spike mode (mean frequency $17 \pm 1 \mathrm{~Hz}$ ), while another group ( 77 out $263,29 \%$ ) fired with an irregular alternation of short bursts and single spikes (mean frequency $6 \pm 0.5 \mathrm{~Hz}$ ). In fifteen neurobiotin-filled FSIs reconstructed with a confocal microscope the different firing patterns did not specifically correspond to diverse morphologies, i.e., bursting, single-spike, or irregularly firing cells displayed dendritic fields which were either relatively spread (180-280 $\mu \mathrm{m}$ of diameter centered at the soma; $n=8)$ or more confined (100-120 $\mu \mathrm{m} ; n=7$; Figure 2B).

All different types were used to analyze membrane properties, connectivity rates, and functional interactions mediated by GJ and GABAergic synapses. Electrical coupling combined with GABAergic synapses was found in 48 out of 146 pairs of FSIs
(33\%) recorded under control conditions (i.e., ACSF $+5 \mu \mathrm{M}$ NBQX; Figure 2C, left panel). Electrical synapses alone were detected in $18 \%$ of total pairs (23\% bidirectionally and $77 \%$ unidirectionally). Another $9 \%$ of total pairs (13 out of 146) were connected via GABAergic synapses only (31\% bidirectionally and 69\% unidirectionally), while the remaining $40 \%$ were unconnected. The somata of pairs that were connected through any of the three patterns described above (i.e., GJ + GABA; GJ alone; GABA alone) were relatively close to each other (82 \pm $2 \mu \mathrm{m})$, whereas pairs with somata separated by larger distances (up to $800 \mu \mathrm{m}$; average $179 \pm 15 \mu \mathrm{m}$ ) were mostly unconnected (Figure 2C, right panel). Thus, FSIs were highly connected to their proximal neighbors but not to more distant cells. Properties of GJ-mediated electrical coupling are shown in Figure 3A. Upon 
injection of hyperpolarizing current steps in one of two simultaneously recorded cells both interneurons responded with voltage deflections corresponding to a CC of $0.06 \pm 0.003(n=$ 74 pairs), yielding a mean Gc of $1.3 \pm 0.1 \mathrm{nS}$ (see Methods). $\mathrm{CC}$ and Gc values were usually asymmetrical within each pair (i.e., $\mathrm{CC}_{\mathrm{FSI} 1 \rightarrow \mathrm{FSI} 2} \neq \mathrm{CC}_{\mathrm{FSI} 2 \rightarrow \mathrm{FSI} 1}$ ). The average smaller value across all pairs was significantly different from the average larger value $\left(\mathrm{CC}_{\text {small }} 0.045 \pm 0.005, \mathrm{CC}_{\text {large }} 0.075 \pm 0.008, n=68, p<\right.$ 0.05 , paired $t$-test; $\mathrm{Gc}_{\text {small }} 1.2 \pm 0.1 \mathrm{nS}, \mathrm{Gc}_{\text {large }} 1.7 \pm 0.2 \mathrm{nS}, n=$ $68, p<0.05$, paired $t$-test). When suprathreshold depolarizing current pulses were injected in $\mathrm{FSI}_{1}$ to induce firing of AP trains, $\mathrm{FSI}_{2}$ responded with arrays of fast spikelets which were timelocked to AP in $\mathrm{FSI}_{1}$. Spikelet properties were measured during presynaptic AP trains at a frequency of $30-40 \mathrm{~Hz}$ in the presence of the $\mathrm{GABA}_{\mathrm{A}}$ receptor antagonist gabazine $(10 \mu \mathrm{M})$. Spikelets were composed by a small, relatively fast depolarizing phase having a mean peak amplitude and time-to-peak of $0.41 \pm 0.04 \mathrm{mV}$ and $1.44 \pm 0.1 \mathrm{~ms}$, respectively, followed by a slower hyperpolarizing trough (mean amplitude and time-to-trough: $0.53 \pm$ $0.06 \mathrm{mV}$ and $10.9 \pm 0.6 \mathrm{~ms}$, respectively). The spikelet positive peak followed the presynaptic spike peak by a delay of $0.9 \pm$ $0.1 \mathrm{~ms}$. Spikelets were reliably evoked by every individual AP across lengthy periods of firing. Temporal summation of presynaptic AP AHPs propagated in a low-pass filtered form to the coupled FSI so that a net $\mathrm{V}_{\mathrm{m}}$ hyperpolarization occurred within the first $100 \mathrm{~ms}$ (mean $73 \pm 3 \mathrm{~ms}, n=38$ pairs) from the negative peak of the first spikelet evoked at the onset of a presynaptic AP train (Figure 3A). Temporal summation was absent at frequencies $<25 \mathrm{~Hz}$, but steeply rose to a maximum in a range between 60 and $120 \mathrm{~Hz}$.

To investigate the interplay between firing activities of FSI pairs connected via electrical and/or chemical synapses, we depolarized $\mathrm{V}_{\mathrm{m}}$ in current-clamp mode to near-threshold values by injecting steady DC ( $\mathrm{I}_{\mathrm{inj}}$ : $\left.300-500 \mathrm{pA}\right)$ in one of the two cells $\left(\mathrm{FSI}_{2}\right.$ in Figure 3B) in order to elicit AP firing at a relatively low frequency $(6.2 \pm 1.3 \mathrm{~Hz}, n=48$ pairs $)$. When $\mathrm{FSI}_{1}$ was also injected with short, repetitive suprathreshold current pulses (200-500 ms, 600 pA), charge coupling through GJ induced a small additional depolarization in $\mathrm{FSI}_{2}$ which in turn increased significantly its firing frequency in correspondence of each current step $(30.1 \pm 2 \mathrm{~Hz}, n=48$ pairs, $p<0.001$, paired-sample $t$-test; Figure $3 \mathrm{~B}$, right). When $\mathrm{FSI}_{1}$ stimulation was ceased, the mean firing frequency in $\mathrm{FSI}_{2}$ returned to an average value similar to that preceding the stimuli $(7.4 \pm 2.0 \mathrm{~Hz}, n=48$ pairs, $p>0.05$, paired-sample $t$-test). Notably, as each of the current pulses was set off the post-firing AHP induced in $\mathrm{FSI}_{1}$ (average 1.3 $\pm 0.2 \mathrm{mV}$ ) was matched by an AHP in $\mathrm{FSI}_{2}$ (average $1.9 \pm 0.3 \mathrm{mV}$; Figure 3C) which was often sufficient to cause a transient pause in firing. Thus, the simultaneous occurrence of a depolarizing drive followed by an AHP in both cells contributed to entrain them into a pattern of firing co-activity. To test the involvement of electrical synapses in pair co-activation we perfused slices with the GJ blocker carbenoxolone (CBX, $200 \mu \mathrm{M}$; Figure 3D) for 30-60 min. In order to prevent unspecific effects by CBX (Tovar et al., 2009), recordings were performed in the presence of AMPA- and $\mathrm{GABA}_{\mathrm{A}}$ receptors antagonists NBQX $(5 \mu \mathrm{M})$ and gabazine $(10 \mu \mathrm{M})$, respectively. CBX also affects NMDA receptors
(Chepkova et al., 2008; Tovar et al., 2009); however, these are not expressed in striatal FSIs (Gittis et al., 2010). In our experiments CBX did not significantly change individual spike waveforms (see below) and was preferred to mefloquine (a more specific blocker of Cx36 GJ protein; Cruikshank et al., 2005) because of a relatively faster inhibitory action by $\mathrm{CBX}$ and because long incubations with $25-100 \mu \mathrm{M}$ mefloquine ( $>2 \mathrm{~h}$ ), besides inducing an expected reduction of the $\mathrm{CC}$, caused a block of repetitive AP firing upon prolonged DC injection, precluding the detection of GJ-mediated effects on spike activity (not shown). CBX caused a $64 \pm 4 \%$ reduction in CC with respect to control conditions (Figure 3D, left inset; $n=8, p<0.05$, paired $t$-test). We also recorded unconnected pairs in which no baseline deflection occurred in response to presynaptic current injection (Figure 3D, right inset). As expected, firing co-activation was completely absent either in non-connected pairs or in GJ-connected pairs after CBX perfusion (Figure 3D, right panel).

These data indicate that GJ-coupled FSIs were entrained into episodes of concurrent firing activity paced by the rhythm of the stimulating current pulses injected in one cell. In order to investigate in more detail the relationship between firing trains, we extended the period of supra-threshold current injection (200-600 pA) in both cells up to $1-2 \mathrm{~min}$ in 47 pairs (Figure 4). During spike trains characterized by relatively low firing rates $(2-20 \mathrm{~Hz}$, with each of the two cells firing at the same or nearly equal average frequency), some spikes were tightly synchronized whereas others induced a postsynaptic spikelet followed by a hyperpolarizing phase (SHP; Figure 4A). Cross-correlation analysis revealed two positive peaks around zero lag $(-2.4 \pm 0.5 \mathrm{~ms}$ and $2.2 \pm 0.2 \mathrm{~ms}$, respectively, $n=11)$ and negative troughs at larger lags $(-14.3 \pm 1.3 \mathrm{~ms}$ and $13.7 \pm 2.1$, respectively, $n=11$; Figure 4A, middle panel), indicating that both spike synchronization and inhibition occurred during low-frequency firing activity. The average cross-correlogram in unconnected pairs did not show significant peaks (Figure 4A, right). At higher frequencies, however, AP firing appeared largely inhibited, i.e., spike bursts occurring in one cell were concomitant with silent periods in the other one (Figure 4B). This phenomenon was particularly evident in pairs where one FSI was bursting while the other displayed a regular or irregular single-spike firing mode (Figure 4B). In these pairs, high-frequency bursts $(25-60 \mathrm{~Hz})$ occurring in $\mathrm{FSI}_{1}$ were often highly effective in silencing or slowing the firing activity of $\mathrm{FSI}_{2}$, even when the firing frequency was augmented in the latter by increasing $I_{\text {inj }}$ amplitude (Figure $4 B$, right). Presynaptic bursts were often able to block or interrupt postsynaptic bursts as well (Figure 4C, left), provided that the two cells fired at an intraburst frequency of less than $50-60 \mathrm{~Hz}$. When both cells exceeded a frequency of $50-60 \mathrm{~Hz}$, however, inhibition failed and the firing became mostly synchronous (Figure 4C, right). In any case, a condition for the occurrence of the latter effect was that at least one cell was tonically firing at high frequency, since we did not observe a plain coincidence of burst activity in two bursting FSIs even when the intra-burst frequency exceeded $50-60 \mathrm{~Hz}$ in both cells.

In order to quantify the inhibitory effect mediated by GJ on AP firing, we computed the Pearson Mean Firing Rate coefficient (PMFR; see Methods) for pairs of spike series 
A

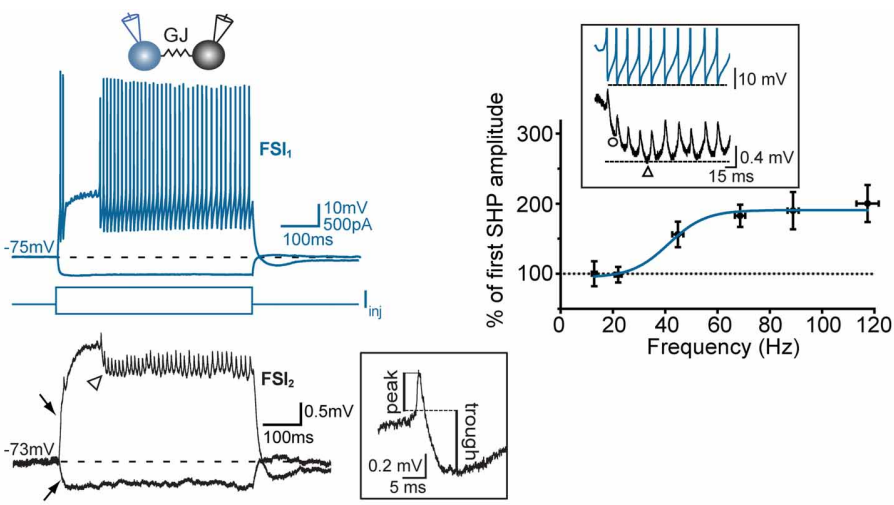

B

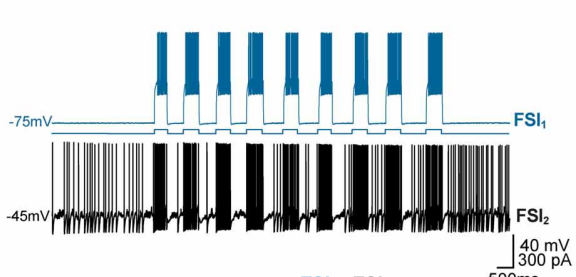

C
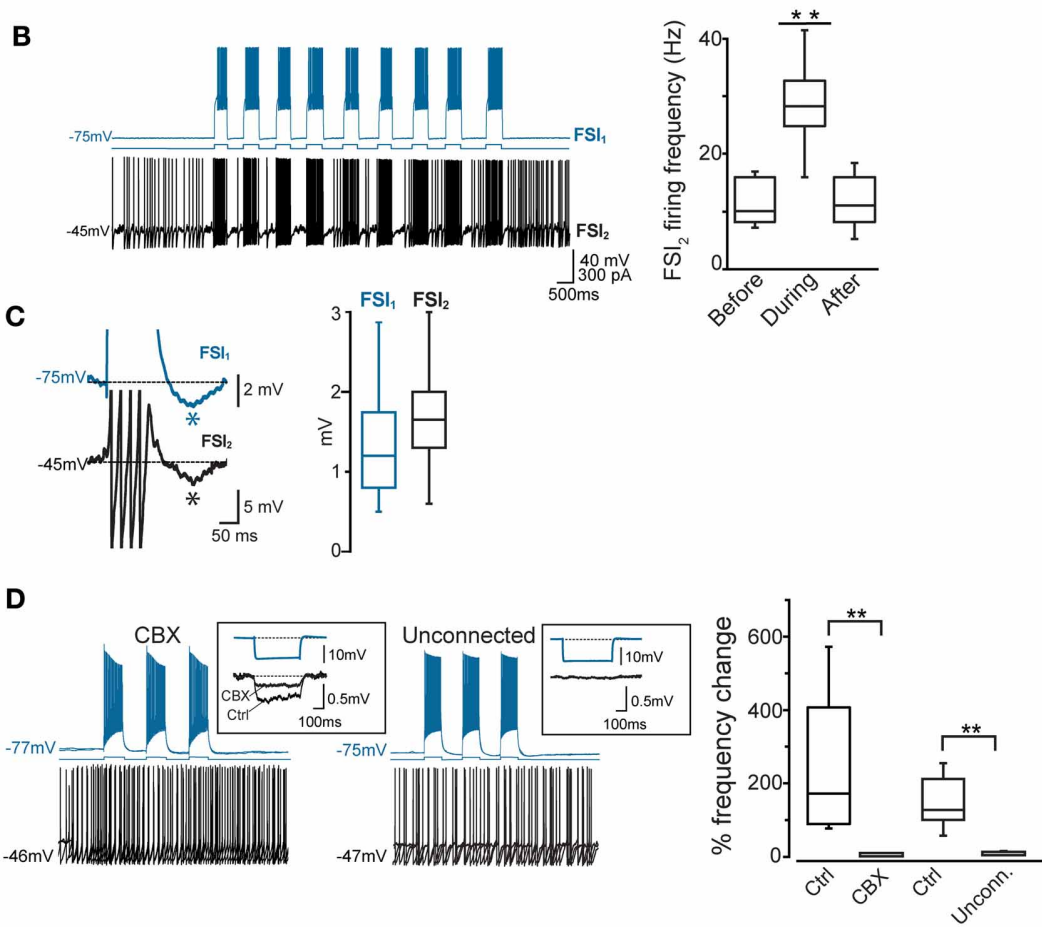

FIGURE 3 | Coordination of firing activity in electrically connected FSIs. (A) Top, voltage responses of $\mathrm{FSI}_{1}$ during injection of hyper- and depolarizing current steps ( -300 and $+400 \mathrm{pA}$, respectively, $500 \mathrm{~ms}$ ). Bottom, passive responses mediated by $\mathrm{GJ}$ in $\mathrm{FSI}_{2}$ (arrows). Spikelets were elicited in $\mathrm{FSI}_{2}$ during AP firing in $\mathrm{FSI}_{1}$. Note temporal summation of spikelet hyperpolarizing potential (SHP, arrowhead). The inset shows an individual spikelet with reference points used for calculating peak and trough amplitudes. Top right panel, frequency-dependence of SHP temporal summation. Each data point represents the average ratio between the amplitude of the most hyperpolarized SHP (marked by a triangle in the inset lower trace) and the amplitude of the first SHP in the array (circle). To reduce data scattering, ratios were grouped within $15-35 \mathrm{~Hz}$ spanning segments along the $x$-axis (each segment contained 10-15 data points from a total of 38 pairs). A Boltzmann fit of the data set (blue line; see Methods) yielded the following parameters: $\mathrm{A} 1=0.95 \pm 0.2, \mathrm{~A} 2=1.9 \pm 0.1, x_{0}=41.2 \pm 5.4 \mathrm{mV}$, and $d x=7.0 \pm 6.3$. Note how spikelet maximal summation was delayed with respect to maximal summation of presynaptic spike AHPs (upper trace in the inset). (B) Left, example of transient, repetitive firing entrainment of two electrically connected FSIs (see text for details). Right, summary of average postsynaptic firing frequencies before, during, and after the injection of current pulses in $\mathrm{FSI}_{1}$ ( $n=48$ pairs, ${ }^{* *} p<0.01$, paired-sample $t$-test).

(C) Left, magnified view of individual burst events occurring in two FSIs in response to a suprathreshold current pulse injected in $\mathrm{FSI}_{1}$. Action potential firing was graphically removed in $\mathrm{FSI}_{1}$ and partially truncated in $\mathrm{FSI}_{2}$ in order to emphasize after-hyperpolarization (AHP, marked by asterisks). Right, summary box plots of AHP amplitudes in $\mathrm{FSI}_{1}$ and $\mathrm{FSI}_{2}$. (D) Lack of firing co-activation in response to injection of suprathreshold current pulses in $\mathrm{FSI}_{1}$ (blue) either in the presence of $200 \mu \mathrm{M}$ carbenoxolone (CBX) or in unconnected pairs. Insets show $\mathrm{V}_{\mathrm{m}}$ changes in $\mathrm{FSI}_{2}$ in response to a hyperpolarizing current pulse $(-300 \mathrm{pA}, 500 \mathrm{~ms})$ injected in $\mathrm{FSI}_{1}$. The box chart on the right shows a statistical summary relative to frequency changes during current injection in $\mathrm{FSI}_{2}$ in control conditions, after bath application of $\mathrm{CBX}\left(n=8,{ }^{* *} p<0.01\right.$, paired $t$-test) and in connected vs. unconnected pairs (** $p<0.01$, unpaired $t$-test, $n=12$ ).

FSI pairs were often connected through GABAergic synapses (see Figures 5A,B), therefore one might attribute the fast AP inhibition to a GABA-mediated inhibitory effect. However, in GJ + GABA connected pairs we found that spike inhibition remained 

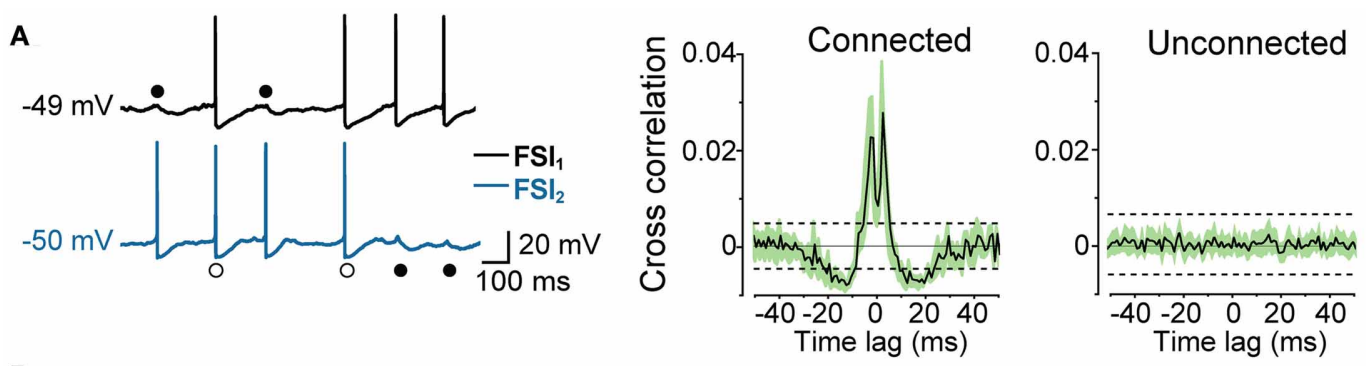

B

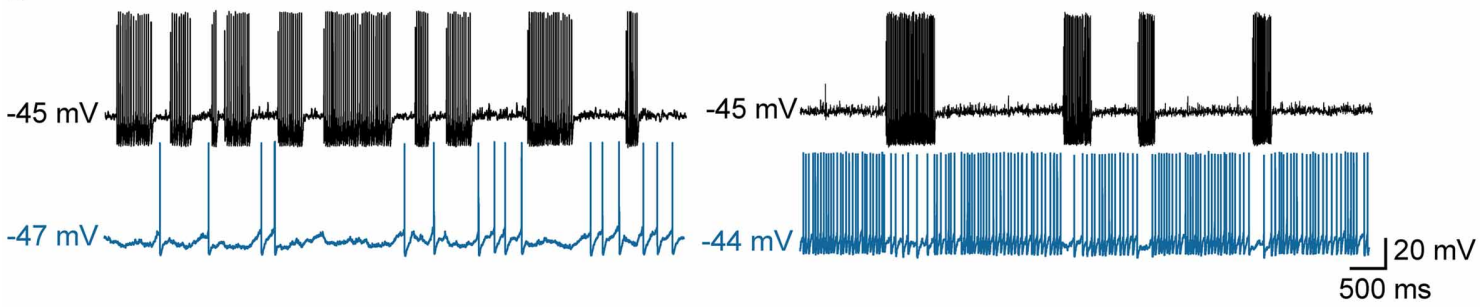

C
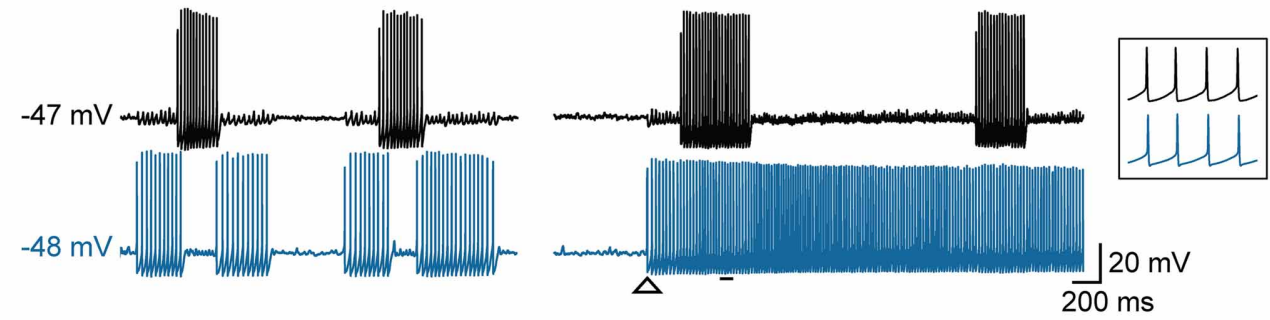

D
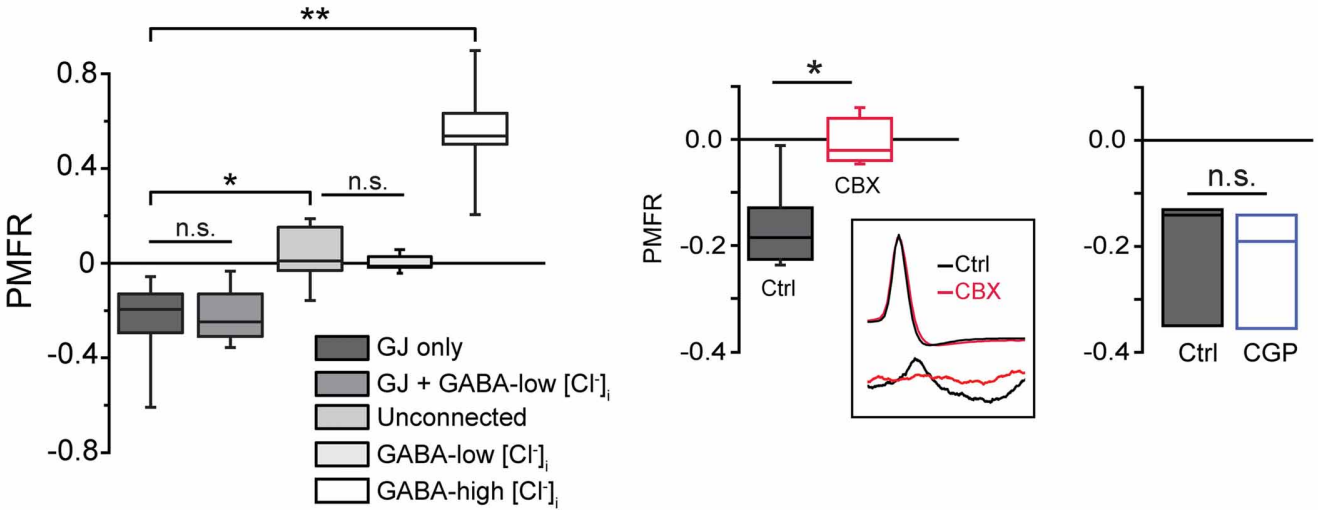

E
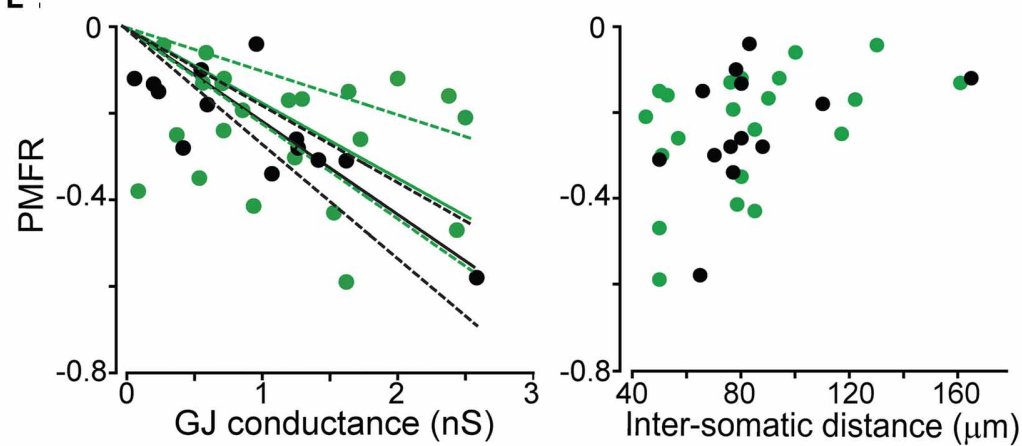

FIGURE 4 | Continued 


\section{FIGURE 4 | GJ mediate inhibition in coupled interneurons at}

high-frequency presynaptic spike firing. (A) In order to induce prolonged episodes of firing activity at low frequency (in this case $8 \mathrm{~Hz}$ in both cells), two electrically connected FSIs (without GABAergic synapses) were injected with a relatively small suprathreshold DC (200-250 pA) through the recording electrodes for 1-2 $\mathrm{min}$. Two-second recording segments are shown here starting 5-10s after the onset of current injection. Left, individual spikes either elicited an AP (white circles) or a spikelet (black circles) in the paired FSI. Right, average cross-correlograms for connected and unconnected pairs ( $n=11$ and 6, respectively). Shaded areas and dashed lines represent s.e.m. and average confidence intervals equal to two standard deviations of the spike trains, respectively $(\mathbf{B})$ Left, burst-like firing episodes in $\mathrm{FSI}_{1}\left(\mathrm{I}_{\text {inj }}\right.$ : $430 \mathrm{pA}$; intra-burst frequency: $38 \mathrm{~Hz}$ ) were associated with silent periods in $\mathrm{FSI}_{2}$ when the latter was stimulated just above firing threshold $\left(\mathrm{l}_{\text {inj }}: 310 \mathrm{pA}\right.$; average spike frequency: $1.8 \mathrm{~Hz}$ ). Right, in the same pair, $\mathrm{FSI}_{2}$ was injected with stronger DC $\left(\mathrm{I}_{\text {inj }}: 370 \mathrm{pA}\right)$ to increase the firing frequency $(\sim 20 \mathrm{~Hz})$. Spike trains in $\mathrm{FSI}_{1}$ were still able to induce a reduction of firing activity in $\mathrm{FSI}_{2}$.
(C) Left, spike inhibition resulted in alternated burst firing in two electrically connected FSIs. Right, in the same pair, the inhibitory effect was overrun by a strong increase in firing frequency in $\mathrm{FSI}_{2}$ (from 29 to $52 \mathrm{~Hz}$; linj was increased from 380 to 430 pA at the instant indicated by the arrowhead). The inset shows synchronous spikes in a magnified time window indicated by the horizontal bar. (D) Left, box-plot summary of Pearson's mean firing rate coefficient values (PMFR) for connected and unconnected FSI pairs

$\left({ }^{*} p<0.05,{ }^{* *} p<0.01\right.$, n.s.: not significant at $p>0.05$, unpaired $t$-test). Right, box plot summarizing the effect of CBX $(200 \mu \mathrm{M})$ and CGP52432 $(5 \mu \mathrm{M})$ relative to paired controls $(* p<0.05, n=5$; n.s.: $p>0.05, n=3$, Wilcoxon signed rank test). The inset shows the inhibitory effect of $\mathrm{CBX}$ on spikelet (bottom) but not AP waveforms (top). (E) PMFR values plotted against GJ conductance (left) and inter-somatic distance (right) for pairs connected by GJ-only (green circles) and GJ + GABA (black circles). Data sets are the same as the ones used for box plots in (D). Solid lines are linear regression fits of the respective data sets (dashed lines represent confidence bands at $95 \%$ level). intact after blocking GABAergic currents through bath application of the $\mathrm{GABA}_{\mathrm{A}}$ receptor antagonist gabazine $(10 \mu \mathrm{M}$; see Figure 5C), or in pairs where GABAergic synapses were undetectable (Figure 5D). The mean PMFR value for FSIs connected exclusively through GJ was $-0.23 \pm 0.03(n=22)$, not significantly different from pairs coupled through GJ $+\mathrm{GABA}(p>0.3$, unpaired $t$-test). In unconnected pairs the mean PMFR value was $0.01 \pm 0.05$ (corresponding to uncorrelated firing activity), significantly different from both GJ-only and GJ + GABA values $(p<$ $0.05, n=12$, unpaired $t$-test) but not significantly different from the mean PMFR calculated in five pairs connected exclusively through GABAergic synapses $(-0.01 \pm 0.01, p>0.05$, unpaired $t$-test; Figure 4D) confirming that GABAergic IPSPs did not exert substantial inhibition on postsynaptic firing activity in these cells.

To further validate the ability of PMFR analysis to identify a causal relationship between spike series, we tested whether the algorithm could detect positive covariations in synchronous spike trains. To do so, we performed paired recordings using an intracellular solution containing a high $\mathrm{Cl}^{-}$concentration $(114 \mathrm{mM})$, which resulted in a strong rightward shift in the reversal potential for $\mathrm{Cl}^{-}$ions and thus caused GABAergic postsynaptic potentials (PSP) to be depolarizing at near-threshold voltage levels (Supplementary Figure S1A). Under these conditions, GABAergic PSPs triggered AP in the postsynaptic FSI resulting in a tight synchronization of firing (Supplementary Figures S1B-D). The mean PMFR value obtained in pairs recorded in high $\left[\mathrm{Cl}^{-}\right]_{\mathrm{i}}$ conditions was +0.51 $\pm 0.1(n=6)$. This value, representing positively covarying firing rates, was significantly different from all other conditions $(p<0.01$, unpaired $t$-test).

In five other GJ-connected pairs the mean PMFR values before and after bath application of $200 \mu \mathrm{M} \mathrm{CBX}$ were $-0.15 \pm 0.03$ and $0.01 \pm 0.03$, respectively $(n=5, p<0.05$, Wilcoxon signed rank test; Figure 4D). CBX did not significantly alter the AP shape observed during prolonged DC injection (peak amplitude, ctrl: $54 \pm 2 \mathrm{mV}$, CBX: $53 \pm 5 \mathrm{mV}$; spike width, ctrl: $0.6 \pm 0.03 \mathrm{~ms}, 0.7$ $\pm 0.05 \mathrm{~ms}$; AHP amplitude, ctrl: $18 \pm 1 \mathrm{mV}$, CBX: $18 \pm 1 \mathrm{mV}$; $n=10$ cells, $p>0.05$, Wilcoxon signed rank test). Conversely, no postsynaptic spikelets were detected in response to presynaptic AP after CBX application (Figure 4D, inset). Furthermore, the
GABA $_{B}$ antagonist CGP52432 $(5 \mu \mathrm{M})$ did not affect GJ-mediated inhibition (PMFR ctrl: $-0.21 \pm 0.07$, CGP: $-0.23 \pm 0.06, n=3$, $p>0.05$, Wilcoxon signed rank test; Figure 4D).

PMFR values from pairs connected through GJ-only or GJ + GABA were linearly correlated with Gc values (Figure 4E, left; the largest conductance value was used for each pair). Despite some divergence, no significant difference was found among linear fits of the two data sets $(F=1.45, p>0.2$, Origin fit comparison $F$-test), confirming that GABAergic synapses did not substantially contribute to AP inhibition. Furthermore, PMFR values diminished as the inter-somatic distance of recorded cells increased (Figure 4E, right). Although the scarcity of connected pairs at inter-somatic distances larger than $130-150 \mu \mathrm{m}$ prevented an appropriate fit of the data sets, these data suggest that spike decoupling had a similar distance-dependence for both GJ-only and GJ + GABA connected pairs.

Finally, the inhibitory effect by GJ was tested in pairs in which AP firing was induced by injecting poissonian trains of sEPSCs at high frequency $(200-500 \mathrm{~Hz})$ in both cells using dynamicclamp (Figure 6). For each pair the two cells were injected with two uncorrelated sEPSC trains. Trains at higher frequencies $(400-500 \mathrm{~Hz})$ were sufficient to supra-linearly depolarize $\mathrm{V}_{\mathrm{m}}$, while lower frequencies required a relatively small amount of DC injection (50-100 pA) to reach threshold. In both cases, the firing activity was negatively correlated (mean PMFR value in GJconnected FSIs: $-0.15 \pm 0.03$; unconnected FSIs: $0.02 \pm 0.04$, $n=6, p<0.05$, Mann-Whitney U test).

Thus, electrical synapses in FSI pairs induced two apparently opposite effects: (1) a relatively slow membrane potential coupling, which resulted in a modulation of the firing frequency of one FSI in relatively loose coherence with the other, and (2) a fast AP inhibition causing a mutual firing suppression which could persist during prolonged periods of activity at relatively high frequencies. The ability of GJ to dampen AP firing becomes evident by analyzing $\mathrm{V}_{\mathrm{m}}$ changes at subthreshold levels during concurrent firing in electrically connected FSIs. As mentioned earlier, trains of spikelets displayed temporal summation of hyperpolarizing phases (Figure 3A; cf. Galarreta and Hestrin, 2001), suggesting that a net inhibitory drive might account for spike silencing during a presynaptic burst. Indeed, trains of 


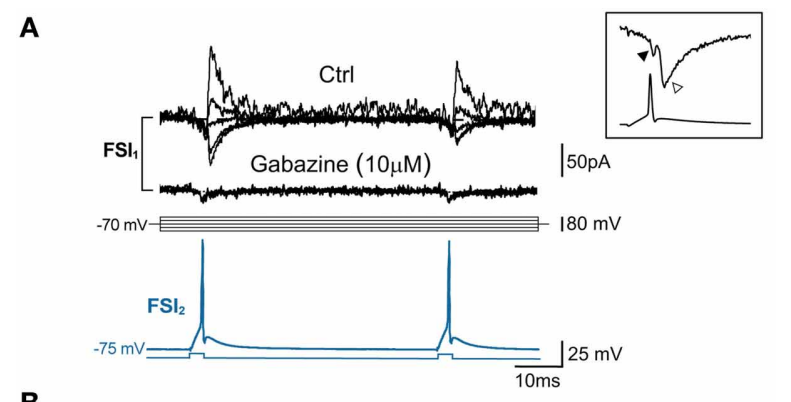

B
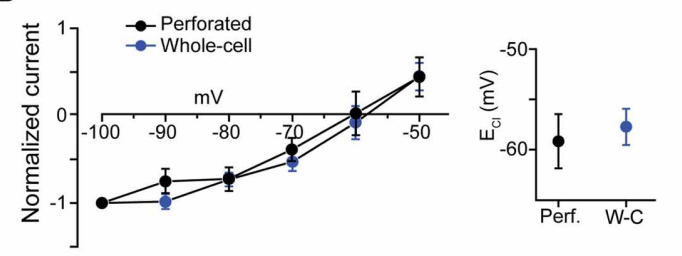

C

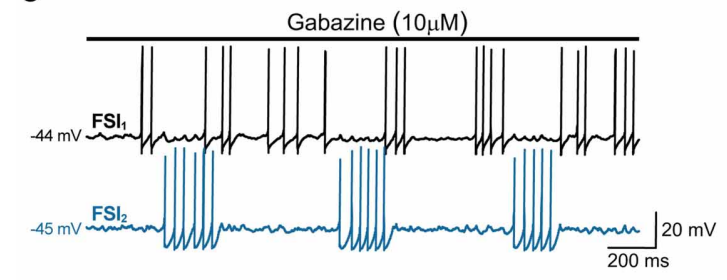

D

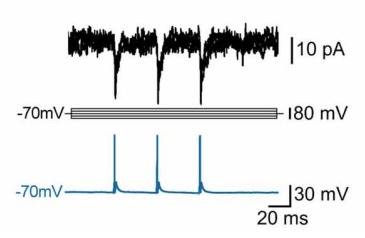

GJ only

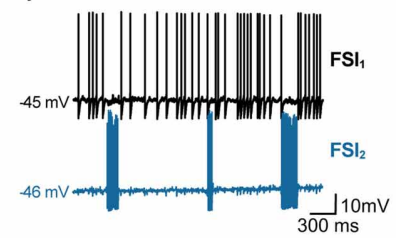

FIGURE 5 | $\mathrm{GABA}_{A}$-mediated fast synaptic transmission is not required for spike inhibition. (A) GABAergic IPSCs (top) evoked in $\mathrm{FSI}_{1}$ at various holding potentials (from -100 to $-20 \mathrm{mV}$, 10-mV steps; middle) in response to two consecutive AP elicited in FSI2 at an interval of $50 \mathrm{~ms}$ (bottom). In pairs connected by GABAergic synapses, mean peak amplitude, $10-90 \%$ rise time, and decay time constant of unitary IPSCs (recorded in voltage-clamp mode at $V_{\text {clamp }}=-90 \mathrm{mV}$ ) were $-70 \pm 14 \mathrm{pA}, 0.6 \pm 0.08 \mathrm{~ms}$, and $3.7 \pm 0.5 \mathrm{~ms}$, respectively, while the mean paired-pulse ratio was $0.85 \pm 0.05$. Bath application of $10 \mu \mathrm{M}$ gabazine for 5-10 min completely blocked the IPSCs, unmasking small GJ-mediated currents. Inset, magnification of $I_{G J}$ (black arrowhead) followed by I IABA (white arrowhead) recorded at $-100 \mathrm{mV}$ in response to an individual presynaptic AP (bottom trace) (B) Left, current-voltage plots of IPSC amplitudes (mean \pm s.e.m.) normalized to average values recorded at $-100 \mathrm{mV}$. Blue and black traces represent data obtained with whole-cell $(n=15)$ and perforated patch recordings $(n=4)$, respectively. Right, summary plot of average reversal potentials for $\mathrm{Cl}^{-}$ions $\left(E_{\mathrm{Cl}}\right)$ obtained with perforated patch $(-59.6 \pm 3 \mathrm{mV}, n=4)$ and whole-cell recordings $(-58.2 \pm 2 \mathrm{mV} ; n=15)$. The theoretical $E_{\mathrm{Cl}}$ calculated using the Nernst equation in our experimental conditions was $-59 \mathrm{mV}$ (see Methods). (C) Example of spike series in which inhibition is still evident during application of $10 \mu \mathrm{M}$ gabazine. (D) Left, voltage-clamp recordings of spikelets alone (i.e., without superimposed IPSCs) at various command potentials in response to three presynaptic AP (blue trace) in a pair connected by GJ, but not GABAergic synapses. Right, mutual inhibition of firing activity recorded in the same pair.

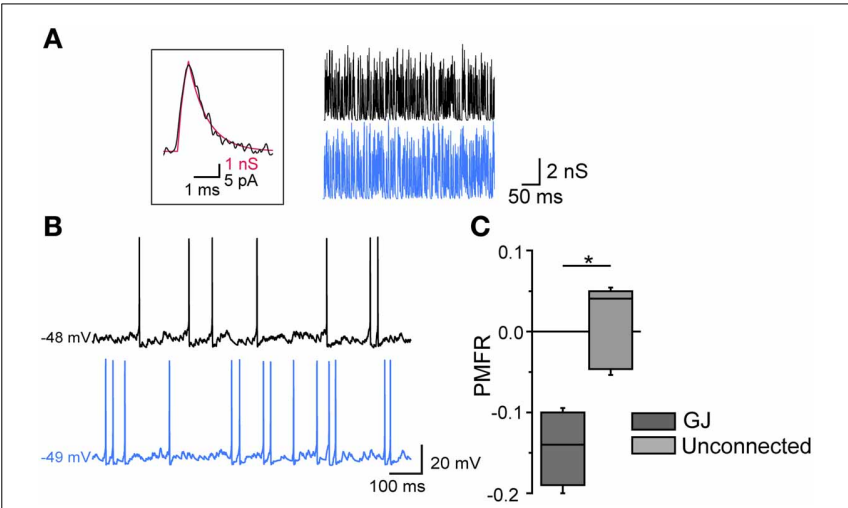

FIGURE 6 | Gap-junction-mediated firing inhibition in FSI pairs stimulated by arrays of sEPSCs in dynamic-clamp configuration.

(A) Left, unitary sEPSC conductance (red trace) superimposed to a unitary spontaneous EPSC (black trace) recorded in FSIs in voltage-clamp mode $\left(V_{\text {clamp }}=-70 \mathrm{mV}\right.$; average of 30 EPSCs recorded in 6 different FSIs). The EPSC waveform was reversed and scaled to that of the sEPSC in order to show matching kinetics ( $10-90 \%$ rise time: $0.3 \mathrm{~ms} ; \tau_{\text {dec }}$ : $0.85 \mathrm{~ms}$ ). Right, Poisson trains of sEPSCs $(300 \mathrm{~Hz}, 500 \mathrm{~ms}$; segments excerpted from traces lasting 15-20 s) used as dynamic-clamp waveforms. (B) Action potential firing in two GJ-connected FSIs stimulated by sEPSC trains shown in (A) in the presence of $10 \mu \mathrm{M}$ gabazine. (C) Summary of PMFR values for GJ-connected and unconnected FSI pairs $\left(n=6,{ }^{*} p<0.05\right.$, Mann-Whitney U test).

spikelets evoked at near-threshold potentials induced an average $\mathrm{V}_{\mathrm{m}}$ hyperpolarization with respect to a preceding time window in which both cells were silent $(-48 \pm 1 \mathrm{mV}$ vs. $-46 \pm 1 \mathrm{mV}$, respectively, $n=22, p<0.05$, paired $t$-test, pairs; Figure 7A). Similarly, the mean presynaptic $\mathrm{V}_{\mathrm{m}}$ level during a train of APs was significantly more hyperpolarized than the $\mathrm{V}_{\mathrm{m}}$ level preceeding the first $\mathrm{AP}(-51 \pm 1 \mathrm{mV}$ vs. $-47 \pm 1 \mathrm{mV}$, respectively, $n=22, p<0.05$, paired $t$-test). Conversely, in unconnected pairs the average postsynaptic subthreshold potential did not change significantly during presynaptic bursts $(-47 \pm 1 \mathrm{mV}$ vs. $-47 \pm 1 \mathrm{mV}$, respectively, $n=10$ pairs, $p>0.05$, paired $t$ test, Figure 7B). Presynaptic mean $V_{m}$ levels before and after AP trains were $-45 \pm 1 \mathrm{mV}$ vs. $-50 \pm 1 \mathrm{mV}$, respectively $(n=$ $10, p<0.05$, paired $t$-test). Thus, GJ-mediated propagation of presynaptic AHPs into the postsynaptic cell induced a small yet consistent hyperpolarization which was sufficient to decrease the firing probability by turning $\mathrm{V}_{\mathrm{m}}$ away from the firing threshold (averaging $-42 \pm 1 \mathrm{mV}, n=30$ cells). To further investigate the hyperpolarizing effect of GJ we used pharmacological manipulations aimed at increasing the spikelet peak and reducing its trough amplitude in order to reverse the ratio between the two phases (Figure 8). Bath application of TEA at low concentration (1 mM) in the presence of $10 \mu \mathrm{M}$ gabazine blocked Kv3-dependent AP repolarizing phase in GJ-connected FSI pairs (Erisir et al., 1999; Lien and Jonas, 2003). TEA induced a significant increase in both AP width (ctrl: $0.43 \pm 0.04 \mathrm{~ms}$, TEA: $0.8 \pm 0.1 \mathrm{~ms}, n=6$ pairs, $p<0.05$, paired $t$-test) and amplitude (ctrl: $57 \pm 4$ mV, TEA: 68 $\pm 4 \mathrm{mV}, p<0.05$ ), and a significant decrease in AHP amplitude (ctrl: $16 \pm 2 \mathrm{mV}$, TEA: $8 \pm 1 \mathrm{mV}, p<0.01$ ). These changes were reflected in the postsynaptic spikelet as a significant increase in 


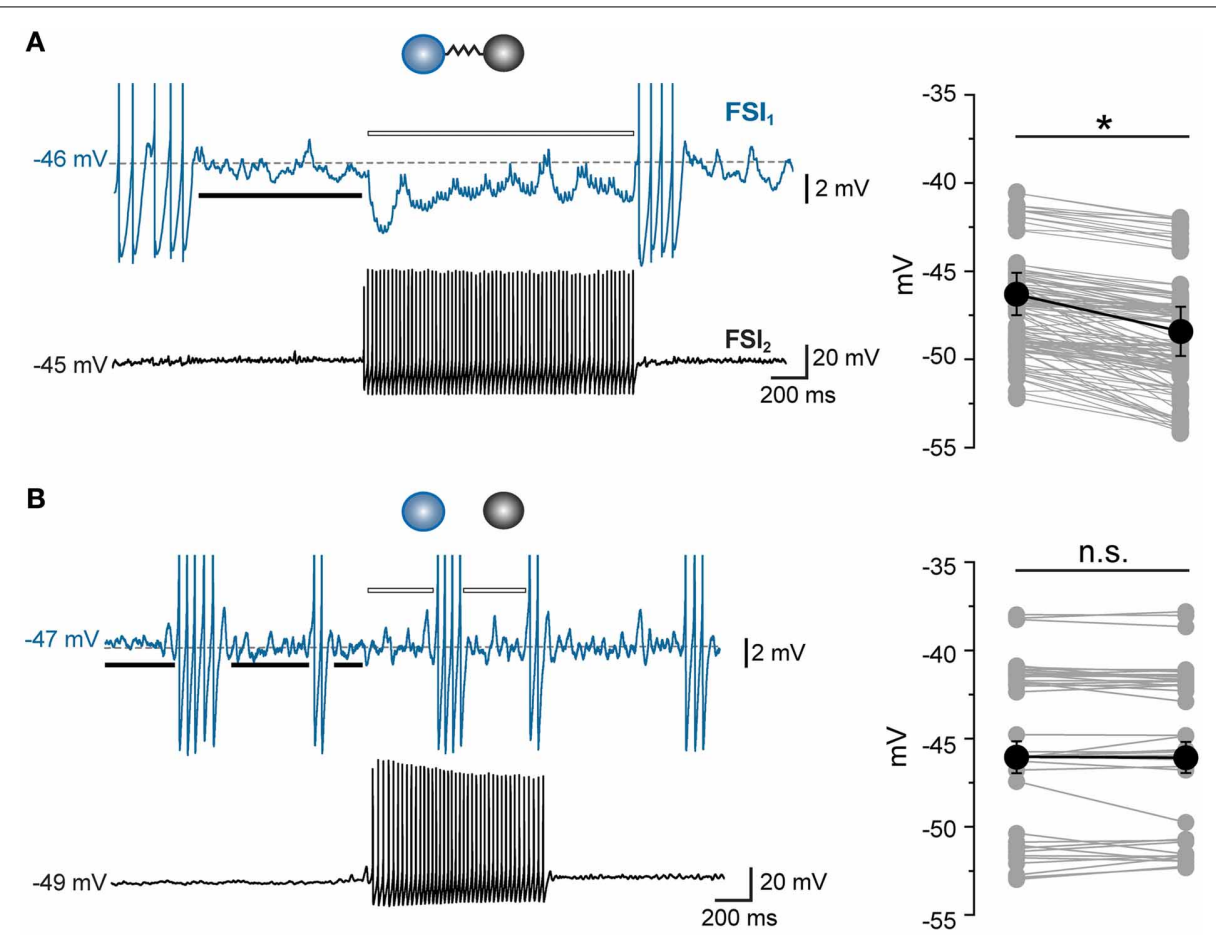

FIGURE 7 | Gap junctions prevent postsynaptic firing by inducing $V_{m}$ hyperpolarization at near-threshold levels. (A) Example of a GJ-only connected pair in which $\mathrm{FSI}_{1}$ displayed an array of subthreshold spikelets in response to a train of $\mathrm{AP}$ occurring in $\mathrm{FSI}_{2}$ during $\mathrm{DC}$ injection in both cells (380 and $405 \mathrm{pA}$, respectively, 20s). The average $\mathrm{V}_{\mathrm{m}}$ value of $\mathrm{FSI}_{1}$ during the spikelet barrage was measured throughout the duration of $\mathrm{FSI}_{2}$ burst (indicated by the white bar) and compared to the average $V_{m}$ value before the burst onset (black bar). Right, summary of average $V_{m}$ values measured in $\mathrm{FSI}_{1}$ before (left column) and during (right column) barrages of 4-8 spikelets occurring in response to AP burst in $\mathrm{FSI}_{2}$. Mean $V_{m} \pm$ s.e.m. values were significantly different in the two conditions ( $n=22$ pairs, $* p<0.05$, paired $t$-test). (B) Same experiment as in (A), but with non-connected FSIs. Mean $\mathrm{V}_{\mathrm{m}} \pm$ s.e.m. in $\mathrm{FSI}_{1}$ did not change significantly during a burst in $\mathrm{FSI}_{2}(n=10$ pairs, $p>0.05$, paired $t$-test). Transient changes in $\mathrm{V}_{\mathrm{m}}$ corresponding to AP firing were excluded from measurements. the ratio between peak and trough amplitudes (ctrl: $0.8 \pm 0.1$, TEA: $3.5 \pm 1.0, p<0.01$, paired $t$-test; Figures $8 \mathrm{~A}, \mathbf{B})$. The CC did not change after TEA application (ctrl: $0.06 \pm 0.01$, TEA: $0.05 \pm 0.01, p>0.05$ paired $t$-test). After blocking Kv3 channels, the spike inhibitory effect was reversed as FSI pairs mainly fired AP in synchrony (PMFR ctrl: $-0.16 \pm 0.04$, TEA: $0.2 \pm 0.08$, $n=6, p<0.05$, paired $t$-test; Figures 8C,D). These data demonstrate that the relatively slow hyperpolarizing phase of spikelets, favored by GJ low-pass filtering properties at the expense of the early fast depolarizing phase, is responsible for a net $V_{m}$ hyperpolarization in response to presynaptic $\mathrm{AP}$ and causes inhibition of postsynaptic firing in GJ-connected FSI pairs.

\section{DISCUSSION}

We investigated the role of GJ in shaping the firing activity of electrically connected FSI pairs recorded in mouse neostriatal slices. Whereas individual APs were either synchronized or inhibited at relatively low firing frequencies $(2-20 \mathrm{~Hz})$, a strong inhibition of AP firing prevailed during presynaptic burst activity at an intra-burst frequency of $25-60 \mathrm{~Hz}$ and postsynaptic firing (either tonic or burst-like) at less than $50 \mathrm{~Hz}$. In pairs composed by two bursting FSIs, GJ often induced burst alternation. In parallel, GJ promoted a relatively slow coupling of membrane potential which allowed the entrainment of connected FSIs into a loosely coordinated firing activity. The magnitude of the AP inhibitory effect was proportional to the amplitude of Gc and was mediated by the hyperpolarizing phase of postsynaptic spikelets. Spike inhibition persisted in the presence of the $\mathrm{GABA}_{\mathrm{A}}$-receptor antagonist gabazine, suggesting that, although they might partially contribute to the effect, fast GABAergic synapses are not necessarily required for the repetitive inhibition of firing activity.

Gap junctions have been shown to induce spike synchrony between cell pairs (including $\mathrm{PV}^{+}$-FSIs similar to the striatal ones described here) in several brain regions (Galarreta and Hestrin, 1999; Gibson et al., 1999; Mann-Metzer and Yarom, 1999; Tamas et al., 2000; Hormuzdi et al., 2001; Szabadics et al., 2001; Traub et al., 2001; Landisman et al., 2002; Bennett and Zukin, 2004; Connors and Long, 2004; Christie et al., 2005; Long et al., 2005; Curti et al., 2012; but see Sippy and Yuste, 2013). Indeed, the occurrence of one or more spikelets at near-threshold potentials may actually trigger AP firing rather than cause inhibition (Mann-Metzer and Yarom, 1999; Curti et al., 2012). Here, however, the fast depolarizing phase of spikelets was not sufficient to reach firing threshold in cells having a relatively low input resistance $\left(\sim 70 \mathrm{M} \Omega\right.$ at resting $\left.\mathrm{V}_{\mathrm{m}}\right)$ such as striatal FSIs. In addition, we did not detect a substantial change in the depolarizing phase of spikelets at near-threshold $\mathrm{V}_{\mathrm{m}}$ as compared to more negative potentials, suggesting that voltage-dependent conductances did not critically contribute to boost the spikelet excitatory phase 


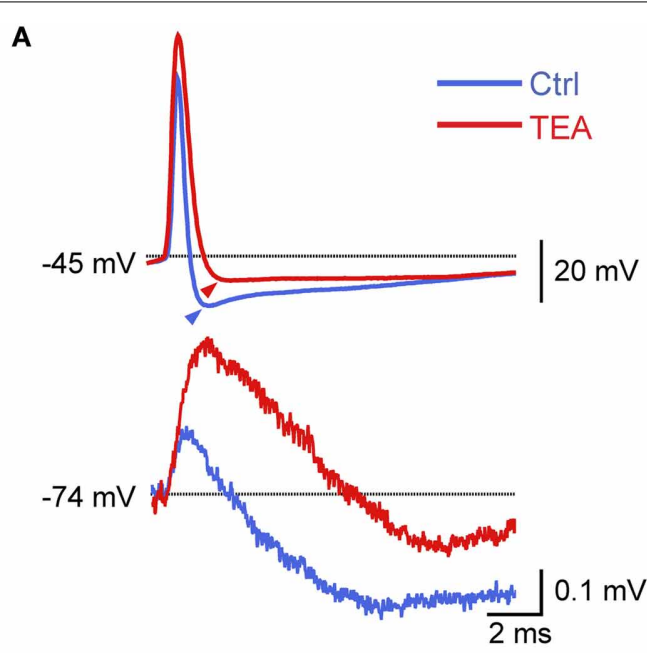

C

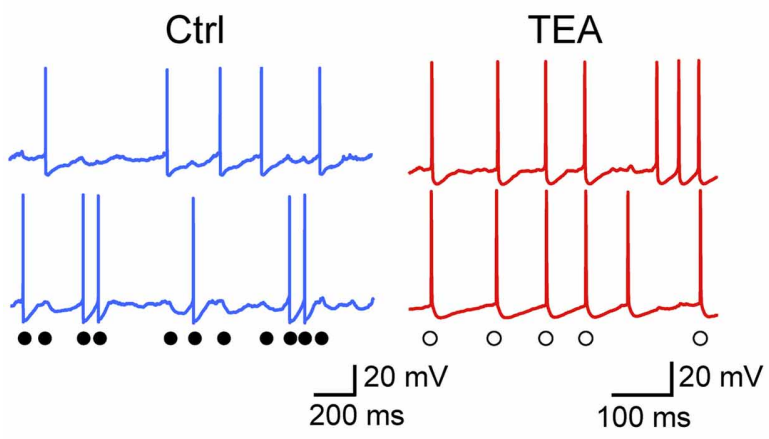

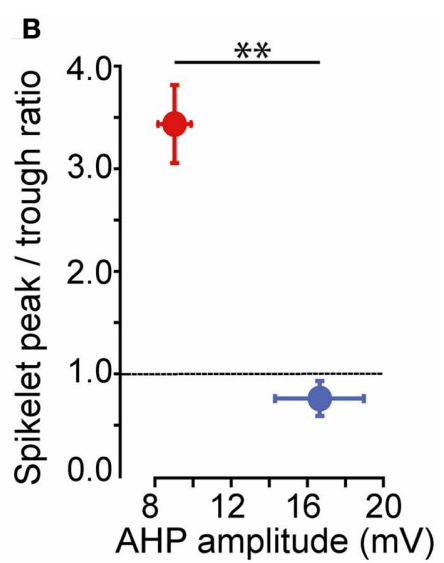

D

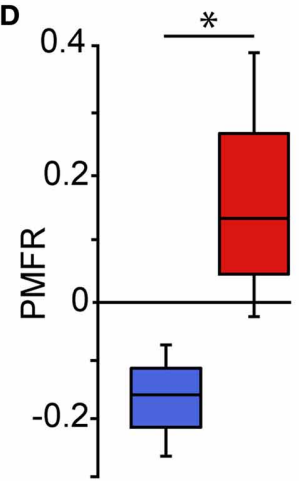

FIGURE 8 | The spikelet hyperpolarizing phase is determinant for GJ-mediated spike inhibition: effect of reducing a Kv3 conductance.

(A) Individual presynaptic AP (top) and postsynaptic spikelets (bottom) recorded in GJ-connected pairs in control conditions and after bath application of $1 \mathrm{mM}$ TEA. Arrowheads indicate AHP peak levels with respect to spike threshold (marked by dotted line). (B) Summary of spikelet peak/trough ratios plotted against AHP amplitudes $(n=6$,
${ }^{* *} p<0.01$, paired $t$-test). (C) Examples of AP trains induced by DC injection (300-400 pA) in ctrl (blue) and after bath application of $1 \mathrm{mM}$ TEA (red). Filled circles indicate spike inhibition in control conditions while empty circles mark synchronized spikes after TEA application. (D) Summary of PMFR coefficients switching from negative (ctrl) to positive (TEA) values according to the change in ratio between spikelet peak and trough amplitudes ( $n=6,{ }^{*} p<0.05$, paired $t$-test). which may result in AP triggering (Mann-Metzer and Yarom, 1999; Curti et al., 2012). Conversely, the slower hyperpolarizing phase provided effective and reliable inhibition, in particular during repetitive presynaptic firing which promoted temporal summation of spikelet AHPs. Pharmacological reduction of the presynaptic AHP resulted in a reversal of the ratio between the spikelet depolarizing peak and hyperpolarizing trough, which was sufficient to cause an overall synchronous spike activity in coupled FSIs. Such effect supports the view that electrical synapses exert spike inhibition by means of a net hyperpolarizing effect associated with temporal summation of spike AHPs propagating through GJ, which is particularly efficient at frequencies above $25 \mathrm{~Hz}$.

Computational studies have predicted anti-phased firing (often in a bi-stable equilibrium with synchronization) in simulated electrically coupled cell pairs or networks (Chow and Kopell, 2000; Lewis and Rinzel, 2003; Nomura et al., 2003; Pfeuty et al., 2003; Mancilla et al., 2007), particularly when spikelets displayed a prevalently hyperpolarizing waveform (Ostojic et al.,
2009; Otsuka and Kawaguchi, 2013). In modeled striatal networks, GJ induced a shunting effect by which two FSIs respond to uncoordinated excitatory input with a reduction in their firing frequency (Hjorth et al., 2009). In the cerebellum, pairs of electrically connected Golgi cells exhibited tightly synchronous firing (Dugue et al., 2009; Vervaeke et al., 2010), which transiently became anti-synchronous after eliciting an excitatory input from the mossy fibers (Vervaeke et al., 2010). Conversely, striatal FSI pairs described here displayed distinct properties in that (1) although both positive and negative spike-to-spike correlations occurred at low firing frequencies, burst activity at frequencies $>25 \mathrm{~Hz}$ was mainly inhibitory over postsynaptic firing (2) both GJ-mediated $V_{m}$ coupling and spike inhibition could be induced in the absence of synaptic stimulation after blocking AMPA and $\mathrm{GABA}_{\mathrm{A}}$ receptors, suggesting that they were exclusively mediated by the interplay between GJ and intrinsic membrane properties, and (3) in robustly coupled pairs spike inhibition persisted throughout all presynaptic firing trains and did not appear to be a transient phenomenon. A clear differential mechanism by 
which GJ promote spike inhibition in the striatum as opposed to other areas remains to be elucidated. In cortical FSIs recorded in the same slices used for striatal experiments, we could detect a similar $\mathrm{V}_{\mathrm{m}}$ coupling but not the spike inhibition effect (our unpublished results). Further work will assess how cell-specific combinations of dendritic morphology, membrane input resistance, ion channel expression, and GJ localization may contribute to critical differences between electrical coupling in striatal FSIs and their counterparts in other brain areas.

The overall lack of inhibitory effects by GABAergic synapses recorded in low $\left[\mathrm{Cl}^{-}\right]_{i}$ was surprising, especially considering the strong GABAergic inhibition exerted by FSIs onto postsynaptic MSNs in slices (Koos and Tepper, 1999; our unpublished observation). Unitary IPSCs recorded in voltage-clamp mode, when present, were usually small in amplitude $(<-40 \mathrm{pA}$ at $-70 \mathrm{mV})$. Although most of our recordings were conducted in whole-cell configuration, a few experiments using perforated-patch revealed a similar reversal potential for GABAergic currents (approximately $-60 \mathrm{mV}$ ), suggesting that the relatively weak IPSCs were not due to an artificially small driving force imposed by our recording conditions. One cannot exclude that the simultaneous activation of multiple presynaptic FSIs may exert some significant compound inhibition over AP firing; in any case, given the observation that inhibition occurred even after blocking $\mathrm{GABA}_{\mathrm{A}}$ receptors with gabazine, we suggest that in adult slices GABAergic synapses may provide inhibitory control of dendritic excitability and integrating properties rather than a direct block of AP firing. The relatively fast kinetics of IPSCs recorded in this study suggest that such integration likely occurs across proximal dendritic segments (Kita et al., 1990).

Results shown in Figure 3B suggest that GJ, by means of subthreshold $\mathrm{V}_{\mathrm{m}}$ coupling, may assist concurrent excitatory inputs to reach firing threshold in neighboring FSIs and promote coherent firing in a local network of connected interneurons. This form of coincidence detection may occur in response to a transient increase in input correlation (Hjorth et al., 2009), e.g., during synchronous transitions to up-states (Blackwell et al., 2003; Gruber et al., 2009; Schulz et al., 2011) or in correspondence of activity strongly correlated to cortical rhythms (Sharott et al., 2012). In contrast, fast AP inhibition provides a reliable and temporally precise uncoupling in FSI firing which in turn may differentially distribute AP-dependent GABAergic feed-forward inhibition onto postsynaptic MSNs. This effect may be scaled up to relatively large local striatal FSI networks, which have been shown to be generally desynchronized using realistic simulations (Hjorth et al., 2009; Humphries et al., 2009) and also to maintain a globally desynchronized state in the network formed by striatal principal cells (Humphries et al., 2009). In vivo, AP inhibition may account for the overall lack of coherent firing activity (Berke, 2008) and the sharp negative cross-correlation peak (Lansink et al., 2010) detected in striatal FSIs of freely moving rats. On the other hand, membrane potential coupling may support transient co-activation of FSIs occurring in association with engagement in a left-right choice task during operant behavior (Gage et al., 2010). A direct detection of $\mathrm{V}_{\mathrm{m}}$ dynamics that regulate FSI excitability would greatly help identify the action of GJ during specific tasks in vivo-although this represents a difficult task to pursue given the scarcity of striatal FSIs, which should be recorded "blindly" using intracellular electrodes (Gruber et al., 2009; Schulz et al., 2011).

Based on model simulations in which suprathreshold DC injection resulted in synchronization of burst-like firing in FSI pairs, Klaus et al. (2011) recently proposed that lack of synchronized firing in extracellularly recorded striatal FSIs in vivo suggests that burst firing activity in these cells is not driven by a sustained $\mathrm{V}_{\mathrm{m}}$ depolarization-such as that induced by neuromodulators like dopamine (Bracci et al., 2002), serotonin (Blomeley et al., 2009), and acetylcholine (Koos and Tepper, 2002) or following activation of mGluR1 (Bonsi et al., 2007) but is rather triggered by a rapid fluctuation of glutamatergic EPSPs. Although this hypothesis cannot be ruled out, our results alternatively suggest that burst firing trains induced by steady current injection do directly inhibit firing activity in an electrically connected cell. Thus, lack of synchronization in vivo does not necessarily imply that firing is driven solely by phasic glutamatergic input and not by a relatively steady, depolarized up-state.

The integrative properties of electrical synapses may be altered under pathological conditions such as dopamine depletiona hallmark of Parkinson's disease-where striatal cell assemblies become entrained into excessively dominant states of synchronization (Jaidar et al., 2010). In fact, morphological and electrophysiological changes occurring in animal models of striatum-related disorders such as Parkinson's disease (Gittis et al., 2011) and dystonia (Gernert et al., 2000), as well as in human Tourette syndrome (Kalanithi et al., 2005), underlie the critical importance of FSIs in controlling the functioning of striatal networks. FSI microcircuits therefore represent a fundamental target for investigating therapeutic strategies against highly incident and debilitating pathologies.

In conclusion, electrical synapses between identified FSIs in mouse dorsal striatum induced a marked, frequency-dependent inhibition of AP firing together with a loose entrainment into coherent firing through $\mathrm{V}_{\mathrm{m}}$ coupling. Firing inhibition was (1) imposed by the spike AHP propagating through GJ, (2) independent of the presence of GABAergic synapses, and (3) directly proportional to the strength of the GJ coupling. The versatile integrative functions of electrical synapses attribute FSIs a complex role in modulating the activity of local striatal networks.

\section{AUTHOR CONTRIBUTIONS}

Giovanni Russo performed electrophysiological recordings and analyzed data; Thierry R. Nieus designed the spike train analysis, modeled the randomized EPSCs and analyzed data; Silvia Maggi analyzed data; Stefano Taverna designed experiments, analyzed data and wrote the manuscript.

\section{ACKNOWLEDGMENTS}

This work was supported by EU-FP7-Health-2013 (grant 602531) to Stefano Taverna. We thank Dr. Fabio Benfenati for helpful comments on the manuscript and Daniela Cantatore, Riccardo Navone, Roberta Pelizzoli, Mattia Pesce, and Federica Piccardi for technical support. 


\section{SUPPLEMENTARY MATERIAL}

The Supplementary Material for this article can be found online at: http://www.frontiersin.org/journal/10.3389/ fncel.2013.00209/abstract

Figure S1 | Synchronizing effect of depolarizing GABA. (A) I-V plot of GABAergic currents recorded using a high intracellular $\mathrm{Cl}^{-}$solution $(114 \mathrm{mM})$. The dotted line is a linear fit of data points extrapolated to the intersection with the $x$-axis (estimated $E_{C l}=-4 \mathrm{mV}$ ). Inset, individual depolarizing postsynaptic potential (dPSP, black trace) evoked at a $V_{m}$ of $-52 \mathrm{mV}$ by a presynaptic AP (blue trace). (B) Co-activation of AP firing during short pulses of supra-threshold current injected in $\mathrm{FSI}_{2}$. This pair was connected via GABAergic synapses only. Inset: voltage response to a hyperpolarizing current injection in $\mathrm{FSI}_{2}$ evoked no $\mathrm{V}_{\mathrm{m}}$ coupling in $\mathrm{FSI}_{1}$, i.e., the two cells were not connected through electrical synapses. Differently from pairs connected via GJ, here AP synchronization was induced by GABAergic depolarizing PSPs. (C) During firing activity evoked by DC injection (300-400 pA), individual spikes were tightly synchronized (top). Firing activity became mostly asynchronous after blocking $\mathrm{GABA}_{\mathrm{A}}-\mathrm{Rs}$ via bath application of $10 \mu \mathrm{M}$ gabazine. (D) Cross-correlograms relative to traces in (C). A significant peak at $\sim 3 \mathrm{~ms}$ was visible in control conditions but not after application of gabazine.

\section{REFERENCES}

Bartos, M., Vida, I., Frotscher, M., Meyer, A., Monyer, H., Geiger, J. R., et al. (2002). Fast synaptic inhibition promotes synchronized gamma oscillations in hippocampal interneuron networks. Proc. Natl. Acad. Sci. U.S.A. 99, 13222-13227. doi: 10.1073/pnas.192233099

Bennett, M. V., and Zukin, R. S. (2004). Electrical coupling and neuronal synchronization in the mammalian brain. Neuron 41, 495-511. doi: 10.1016/S08966273(04)00043-1

Berke, J. D. (2008). Uncoordinated firing rate changes of striatal fast-spiking interneurons during behavioral task performance. J. Neurosci. 28, 10075-10080. doi: 10.1523/JNEUROSCI.2192-08.2008

Berke, J. D. (2011). Functional properties of striatal fast-spiking interneurons. Front. Syst. Neurosci. 5:45. doi: 10.3389/fnsys.2011.00045

Blackwell, K. T., Czubayko, U., and Plenz, D. (2003). Quantitative estimate of synaptic inputs to striatal neurons during up and down states in vitro. J. Neurosci. 23, 9123-9132.

Blomeley, C. P., Kehoe, L. A., and Bracci, E. (2009). Substance P mediates excitatory interactions between striatal projection neurons. J. Neurosci. 29, 4953-4963. doi: 10.1523/JNEUROSCI.6020-08.2009

Bonsi, P., Sciamanna, G., Mitrano, D. A., Cuomo, D., Bernardi, G., Platania, P., et al. (2007). Functional and ultrastructural analysis of group I mGluR in striatal fast-spiking interneurons. Eur. J. Neurosci. 25, 1319-1331. doi: 10.1111/j.14609568.2007.05383.x

Bracci, E., Centonze, D., Bernardi, G., and Calabresi, P. (2002). Dopamine excites fast-spiking interneurons in the striatum. J. Neurophysiol. 87, 2190-2194. doi: 10.1152/jn.00754.2001

Bracci, E., Centonze, D., Bernardi, G., and Calabresi, P. (2003). Voltage-dependent membrane potential oscillations of rat striatal fast-spiking interneurons. J. Physiol. 549, 121-130. doi: 10.1113/jphysiol.2003.040857

Chepkova, A. N., Sergeeva, O. A., and Haas, H. L. (2008). Carbenoxolone impairs LTP and blocks NMDA receptors in murine hippocampus. Neuropharmacology 55, 139-147. doi: 10.1016/j.neuropharm.2008.05.001

Chow, C. C., and Kopell, N. (2000). Dynamics of spiking neurons with electrical coupling. Neural Comput. 12, 1643-1678. doi: 10.1162/0899766003000 15295

Christie, J. M., Bark, C., Hormuzdi, S. G., Helbig, I., Monyer, H., and Westbrook, G. L. (2005). Connexin36 mediates spike synchrony in olfactory bulb glomeruli. Neuron 46, 761-772. doi: 10.1016/j.neuron.2005.04.030

Connors, B. W., and Long, M. A. (2004). Electrical synapses in the mammalian brain. Annu. Rev. Neurosci. 27, 393-418. doi: 10.1146/annurev.neuro.26.041002. 131128
Cruikshank, S. J., Landisman, C. E., Mancilla, J. G., and Connors, B. W. (2005). Connexon connexions in the thalamocortical system. Prog. Brain Res. 149, 41-57. doi: 10.1016/S0079-6123(05)49004-4

Curti, S., Hoge, G., Nagy, J. I., and Pereda, A. E. (2012). Synergy between electrical coupling and membrane properties promotes strong synchronization of neurons of the mesencephalic trigeminal nucleus. J. Neurosci. 32, 4341-4359. doi: 10.1523/JNEUROSCI.6216-11.2012

Dugue, G. P., Brunel, N., Hakim, V., Schwartz, E., Chat, M., Levesque, M., et al. (2009). Electrical coupling mediates tunable low-frequency oscillations and resonance in the cerebellar golgi cell network. Neuron 61, 126-139. doi: 10.1016/j.neuron.2008.11.028

Ebihara, S., Shirato, K., Harata, N., and Akaike, N. (1995). Gramicidin-perforated patch recording: GABA response in mammalian neurones with intact intracellular chloride. J. Physiol. 484, 77-86.

Erisir, A., Lau, D., Rudy, B., and Leonard, C. S. (1999). Function of specific $\mathrm{K}(+)$ channels in sustained high-frequency firing of fast-spiking neocortical interneurons. J. Neurophysiol. 82, 2476-2489.

Fukuda, T. (2009). Network architecture of gap junction-coupled neuronal linkage in the striatum. J. Neurosci. 29, 1235-1243. doi: 10.1523/JNEUROSCI.441808.2009

Gage, G. J., Stoetzner, C. R., Wiltschko, A. B., and Berke, J. D. (2010). Selective activation of striatal fast-spiking interneurons during choice execution. Neuron 67, 466-479. doi: 10.1016/j.neuron.2010.06.034

Galarreta, M., and Hestrin, S. (1999). A network of fast-spiking cells in the neocortex connected by electrical synapses. Nature 402, 72-75. doi: 10.1038/47029

Galarreta, M., and Hestrin, S. (2001). Electrical synapses between GABA-releasing interneurons. Nat. Rev. Neurosci. 2, 425-433. doi: 10.1038/35077566

Galarreta, M., and Hestrin, S. (2002). Electrical and chemical synapses among parvalbumin fast-spiking GABAergic interneurons in adult mouse neocortex. Proc. Natl. Acad. Sci. U.S.A. 99, 12438-12443. doi: 10.1073/pnas.192159599

Gerfen, C. R., Baimbridge, K. G., and Miller, J. J. (1985). The neostriatal mosaic: compartmental distribution of calcium-binding protein and parvalbumin in the basal ganglia of the rat and monkey. Proc. Natl. Acad. Sci. U.S.A. 82, 8780-8784. doi: 10.1073/pnas.82.24.8780

Gernert, M., Hamann, M., Bennay, M., Loscher, W., and Richter, A. (2000). Deficit of striatal parvalbumin-reactive GABAergic interneurons and decreased basal ganglia output in a genetic rodent model of idiopathic paroxysmal dystonia. J. Neurosci. 20, 7052-7058.

Gibson, J. R., Beierlein, M., and Connors, B. W. (1999). Two networks of electrically coupled inhibitory neurons in neocortex. Nature 402, 75-79. doi: $10.1038 / 47035$

Gittis, A. H., Leventhal, D. K., Fensterheim, B. A., Pettibone, J. R., Berke, J. D., and Kreitzer, A. C. (2011). Selective inhibition of striatal fastspiking interneurons causes dyskinesias. J. Neurosci. 31, 15727-15731. doi: 10.1523/JNEUROSCI.3875-11.2011

Gittis, A. H., Nelson, A. B., Thwin, M. T., Palop, J. J., and Kreitzer, A. C. (2010). Distinct roles of GABAergic interneurons in the regulation of striatal output pathways. J. Neurosci. 30, 2223-2234. doi: 10.1523/JNEUROSCI.487009.2010

Gruber, A. J., Powell, E. M., and O’Donnell, P. (2009). Cortically activated interneurons shape spatial aspects of cortico-accumbens processing. J. Neurophysiol. 101, 1876-1882. doi: 10.1152/jn.91002.2008

Gustafson, N., Gireesh-Dharmaraj, E., Czubayko, U., Blackwell, K. T., and Plenz, D. (2006). A comparative voltage and current-clamp analysis of feedback and feedforward synaptic transmission in the striatal microcircuit in vitro. J. Neurophysiol. 95, 737-752. doi: 10.1152/jn.00802.2005

Hjorth, J., Blackwell, K. T., and Kotaleski, J. H. (2009). Gap junctions between striatal fast-spiking interneurons regulate spiking activity and synchronization as a function of cortical activity. J. Neurosci. 29, 5276-5286. doi: 10.1523/JNEUROSCI.6031-08.2009

Hormuzdi, S. G., Pais, I., Lebeau, F. E., Towers, S. K., Rozov, A., Buhl, E. H., et al. (2001). Impaired electrical signaling disrupts gamma frequency oscillations in connexin 36-deficient mice. Neuron 31, 487-495. doi: 10.1016/S08966273(01)00387-7

Humphries, M. D., Wood, R., and Gurney, K. (2009). Dopamine-modulated dynamic cell assemblies generated by the GABAergic striatal microcircuit. Neural Netw. 22, 1174-1188. doi: 10.1016/j.neunet.2009.07.018

Jaidar, O., Carrillo-Reid, L., Hernandez, A., Drucker-Colin, R., Bargas, J., and Hernandez-Cruz, A. (2010). Dynamics of the Parkinsonian striatal microcircuit: 
entrainment into a dominant network state. J. Neurosci. 30, 11326-11336. doi: 10.1523/JNEUROSCI.1380-10.2010

Kalanithi, P. S., Zheng, W., Kataoka, Y., Difiglia, M., Grantz, H., Saper, C. B., et al. (2005). Altered parvalbumin-positive neuron distribution in basal ganglia of individuals with Tourette syndrome. Proc. Natl. Acad. Sci. U.S.A. 102, 13307-13312. doi: 10.1073/pnas.0502624102

Kita, H., Kosaka, T., and Heizmann, C. W. (1990). Parvalbumin-immunoreactive neurons in the rat neostriatum: a light and electron microscopic study. Brain Res. 536, 1-15. doi: 10.1016/0006-8993(90)90002-S

Klaus, A., Planert, H., Hjorth, J. J., Berke, J. D., Silberberg, G., and Kotaleski, J. H. (2011). Striatal fast-spiking interneurons: from firing patterns to postsynaptic impact. Front. Syst. Neurosci. 5:57. doi: 10.3389/fnsys.2011.00057

Koos, T., and Tepper, J. M. (1999). Inhibitory control of neostriatal projection neurons by GABAergic interneurons. Nat. Neurosci. 2, 467-472. doi: 10.1038/8138

Koos, T., and Tepper, J. M. (2002). Dual cholinergic control of fast-spiking interneurons in the neostriatum. J. Neurosci. 22, 529-535.

Landisman, C. E., Long, M. A., Beierlein, M., Deans, M. R., Paul, D. L., and Connors, B. W. (2002). Electrical synapses in the thalamic reticular nucleus. J. Neurosci. 22, 1002-1009.

Lansink, C. S., Goltstein, P. M., Lankelma, J. V., and Pennartz, C. M. (2010). Fast-spiking interneurons of the rat ventral striatum: temporal coordination of activity with principal cells and responsiveness to reward. Eur. J. Neurosci. 32, 494-508. doi: 10.1111/j.1460-9568.2010.07293.x

Lewis, T. J., and Rinzel, J. (2003). Dynamics of spiking neurons connected by both inhibitory and electrical coupling. J. Comput. Neurosci. 14, 283-309. doi: 10.1023/A:1023265027714

Lien, C. C., and Jonas, P. (2003). Kv3 potassium conductance is necessary and kinetically optimized for high-frequency action potential generation in hippocampal interneurons. J. Neurosci. 23, 2058-2068.

Long, M. A., Jutras, M. J., Connors, B. W., and Burwell, R. D. (2005). Electrical synapses coordinate activity in the suprachiasmatic nucleus. Nat. Neurosci. 8, 61-66. doi: 10.1038/nn1361

Luk, K. C., and Sadikot, A. F. (2001). GABA promotes survival but not proliferation of parvalbumin-immunoreactive interneurons in rodent neostriatum: an in vivo study with stereology. Neuroscience 104, 93-103. doi: 10.1016/S03064522(01)00038-0

Madisen, L., Zwingman, T. A., Sunkin, S. M., Oh, S. W., Zariwala, H. A., Gu, H., et al. (2010). A robust and high-throughput Cre reporting and characterization system for the whole mouse brain. Nat. Neurosci. 13, 133-140. doi: $10.1038 / \mathrm{nn} .2467$

Mallet, N., Ballion, B., Le Moine, C., and Gonon, F. (2006). Cortical inputs and GABA interneurons imbalance projection neurons in the striatum of parkinsonian rats. J. Neurosci. 26, 3875-3884. doi: 10.1523/JNEUROSCI.4439-05.2006

Mancilla, J. G., Lewis, T. J., Pinto, D. J., Rinzel, J., and Connors, B. W. (2007). Synchronization of electrically coupled pairs of inhibitory interneurons in neocortex. J. Neurosci. 27, 2058-2073. doi: 10.1523/JNEUROSCI.2715-06.2007

Mann-Metzer, P., and Yarom, Y. (1999). Electrotonic coupling interacts with intrinsic properties to generate synchronized activity in cerebellar networks of inhibitory interneurons. J. Neurosci. 19, 3298-3306.

Mura, A., Linder, J. C., Young, S. J., and Groves, P. M. (2000). Striatal cells containing aromatic L-amino acid decarboxylase: an immunohistochemical comparison with other classes of striatal neurons. Neuroscience 98, 501-511. doi: 10.1016/S0306-4522(00)00154-8

Nomura, M., Fukai, T., and Aoyagi, T. (2003). Synchrony of fast-spiking interneurons interconnected by GABAergic and electrical synapses. Neural Comput. 15, 2179-2198. doi: 10.1162/089976603322297340

Ostojic, S., Brunel, N., and Hakim, V. (2009). Synchronization properties of networks of electrically coupled neurons in the presence of noise and heterogeneities. J. Comput. Neurosci. 26, 369-392. doi: 10.1007/s10827-008-0117-3

Otsuka, T., and Kawaguchi, Y. (2013). Common excitatory synaptic inputs to electrically connected cortical fast-spiking cell networks. J. Neurophysiol. 110, 795-806. doi: 10.1152/jn.00071.2013

Parthasarathy, H. B., and Graybiel, A. M. (1997). Cortically driven immediate-early gene expression reflects modular influence of sensorimotor cortex on identified striatal neurons in the squirrel monkey. J. Neurosci. 17, 2477-2491.
Pennartz, C. M., and Kitai, S. T. (1991). Hippocampal inputs to identified neurons in an in vitro slice preparation of the rat nucleus accumbens: evidence for feedforward inhibition. J. Neurosci. 11, 2838-2847.

Pfeuty, B., Mato, G., Golomb, D., and Hansel, D. (2003). Electrical synapses and synchrony: the role of intrinsic currents. J. Neurosci. 23, 6280-6294.

Planert, H., Szydlowski, S. N., Hjorth, J. J., Grillner, S., and Silberberg, G. (2010). Dynamics of synaptic transmission between fast-spiking interneurons and striatal projection neurons of the direct and indirect pathways. J. Neurosci. 30, 3499-3507. doi: 10.1523/JNEUROSCI.5139-09.2010

Plotkin, J. L., Wu, N., Chesselet, M. F., and Levine, M. S. (2005). Functional and molecular development of striatal fast-spiking GABAergic interneurons and their cortical inputs. Eur. J. Neurosci. 22, 1097-1108. doi: 10.1111/j.14609568.2005.04303.x

Schulz, J. M., Pitcher, T. L., Savanthrapadian, S., Wickens, J. R., Oswald, M. J., and Reynolds, J. N. (2011). Enhanced high-frequency membrane potential fluctuations control spike output in striatal fast-spiking interneurones in vivo. J. Physiol. 589, 4365-4381. doi: 10.1113/jphysiol.2011.212944

Sharott, A., Doig, N. M., Mallet, N., and Magill, P. J. (2012). Relationships between the firing of identified striatal interneurons and spontaneous and driven cortical activities in vivo. J. Neurosci. 32, 13221-13236. doi: 10.1523/JNEUROSCI.244012.2012

Sippy, T., and Yuste, R. (2013). Decorrelating action of inhibition in neocortical networks. J. Neurosci. 33, 9813-9830. doi: 10.1523/JNEUROSCI.457912.2013

Szabadics, J., Lorincz, A., and Tamas, G. (2001). Beta and gamma frequency synchronization by dendritic gabaergic synapses and gap junctions in a network of cortical interneurons. J. Neurosci. 21, 5824-5831.

Tamas, G., Buhl, E. H., Lorincz, A., and Somogyi, P. (2000). Proximally targeted GABAergic synapses and gap junctions synchronize cortical interneurons. Nat. Neurosci. 3, 366-371. doi: 10.1038/73936

Taverna, S., Canciani, B., and Pennartz, C. M. A. (2007). Membrane properties and synaptic connectivity of fast-spiking interneurons in rat ventral striatum. Brain Res. 1152, 49-56. doi: 10.1016/j.brainres.2007.03.053

Tepper, J. M., Tecuapetla, F., Koos, T., and Ibanez-Sandoval, O. (2010). Heterogeneity and diversity of striatal GABAergic interneurons. Front. Neuroanat. 4:150. doi: 10.3389/fnana.2010.00150

Tovar, K. R., Maher, B. J., and Westbrook, G. L. (2009). Direct actions of carbenoxolone on synaptic transmission and neuronal membrane properties. J. Neurophysiol. 102, 974-978. doi: 10.1152/jn.00060.2009

Traub, R. D., Kopell, N., Bibbig, A., Buhl, E. H., Lebeau, F. E., and Whittington, M. A. (2001). Gap junctions between interneuron dendrites can enhance synchrony of gamma oscillations in distributed networks. J. Neurosci. 21, 9478-9486.

Vervaeke, K., Lorincz, A., Gleeson, P., Farinella, M., Nusser, Z., and Silver, R. A. (2010). Rapid desynchronization of an electrically coupled interneuron network with sparse excitatory synaptic input. Neuron 67, 435-451. doi: 10.1016/j.neuron.2010.06.028

Conflict of Interest Statement: The authors declare that the research was conducted in the absence of any commercial or financial relationships that could be construed as a potential conflict of interest.

Received: 19 August 2013; accepted: 21 October 2013; published online: 14 November 2013.

Citation: Russo G, Nieus TR, Maggi S and Taverna S (2013) Dynamics of action potential firing in electrically connected striatal fast-spiking interneurons. Front. Cell. Neurosci. 7:209. doi: 10.3389/fncel.2013.00209

This article was submitted to the journal Frontiers in Cellular Neuroscience.

Copyright (c) 2013 Russo, Nieus, Maggi and Taverna. This is an open-access article distributed under the terms of the Creative Commons Attribution License (CC BY). The use, distribution or reproduction in other forums is permitted, provided the original author(s) or licensor are credited and that the original publication in this journal is cited, in accordance with accepted academic practice. No use, distribution or reproduction is permitted which does not comply with these terms. 